IMA Journal of Numerical Analysis (2019) Page 1 of 21 doi:10.1093/imanum/drn000

\title{
Convergence analysis of high-order commutator-free quasi-Magnus exponential integrators for non-autonomous linear Schrödinger equations
}

\author{
SERGio BLANES \\ Universitat Politècnica de València, Instituto de Matemática Multidisciplinar, \\ 46022 Valencia, Spain.E-mail: serblaza@imm.upv.es \\ FERNANDO CASAS \\ Universitat Jaume I, IMAC and Departament de Matemàtiques, \\ 12071 Castellón,Spain.E-mail: fernando.casas@uji.es \\ CESÁREo GonZÁlez \\ Universidad de Valladolid, Departamento de Matemática Aplicada, \\ 47011 Valladolid, Spain.E-mail: ome@am.uva.es \\ MECHTHILD THALHAMMER \\ Leopold-Franzens-Universität Innsbruck, Institut für Mathematik, \\ 6020 Innsbruck, Austria. E-mail: mechthild.thalhammer@uibk.ac.at
}

[Received on 2 December 2019]

\begin{abstract}
This work is devoted to the derivation of a convergence result for high-order commutator-free quasiMagnus (CFQM) exponential integrators applied to non-autonomous linear Schrödinger equations; a detailed stability and local error analysis is provided for the relevant special case, where the Hamilton operator comprises the Laplacian and a regular space-time-dependent potential. In the context of non-autonomous linear ordinary differential equations, CFQM exponential integrators are composed of exponentials involving linear combinations of certain values of the associated time-dependent matrix; this approach extends to non-autonomous linear evolution equations given by unbounded operators. An inherent advantage of CFQM exponential integrators over other time integration methods such as RungeKutta methods or Magnus integrators is that structural properties of the underlying operator family are well-preserved; this characteristic is confirmed by a theoretical analysis ensuring unconditional stability in the underlying Hilbert space and the full order of convergence under low regularity requirements on the initial state. Due to the fact that convenient tools for products of matrix exponentials such as the Baker-Campbell-Hausdorff formula involve infinite series and thus cannot be applied in connection with unbounded operators, a certain complexity in the investigation of higher-order CFQM exponential integrators for Schrödinger equations is related to an appropriate treatment of compositions of evolution operators; an effective concept for the derivation of a local error expansion relies on suitable linearisations of the evolution equations for the exact and numerical solutions, representations by the variationof-constants formula, and Taylor series expansions of parts of the integrands, where the arising iterated commutators determine the regularity requirements on the problem data.
\end{abstract}

Keywords: Non-autonomous linear evolution equations, Schrödinger equations, Quantum systems, Time integration methods, Exponential integrators, Magnus integrators, Commutator-free quasi-Magnus exponential integrators, Stability, Local error, Convergence

AMS: 65L05, 65M12, 65J10

(c) The author 2019. Published by Oxford University Press on behalf of the Institute of Mathematics and its Applications. All rights reserved. 


\section{Introduction}

Commutator-free quasi-Magnus exponential integrators. Numerical experiments for nonautonomous linear differential equations confirm the favourable stability and error behaviour of exponential methods based on the Magnus expansion in comparison with classical time integration methods, see for instance Blanes, Casas, Oteo, Ros (2009); Blanes, Moan (2006); Hochbruck, Lu$\mathrm{BICH}(2003)$ and references given therein.

However, in the context of large-scale applications, it is advantageous to avoid the actual computation and storage of commutators. For specific Schrödinger equations, an effective methology is proposed in the recent contribution ISERLES, KROPIELNICKA, SINGH (2019), see also references therein; the required special form of the Hamiltonian permits a substantial simplification of the commutators arising in the Magnus expansion.

In the present work, we continue our study of an alternative approach that applies to a more general class of Hamiltonian systems and Schrödinger equations, respectively; it relies on the replacement of a single (matrix) exponential involving iterated commutators by compositions of (matrix) exponentials and leads to numerical approximations that are potentially superior to those obtained by standard Magnus integrators, see Alvermann, Fehske (2011); Alvermann, Fehske, Littlewood (2012); Bader, Blanes, Kopylov (2018); Blanes, Casas, Thalhammer $(2017,2018)$. We refer to this type of time integration methods as commutator-free quasi-Magnus (CFQM) exponential integrators.

In Blanes, CASAS, Thalhammer $(2017,2018)$, our focus was on the study of CFQM exponential integrators applied to dissipative quantum systems or partial differential equations of parabolic type, respectively; during the development of these contributions, we also performed numerical tests for Hamiltonian systems and Schrödinger equations. Our comparisons confirmed the excellent behaviour of CFQM exponential integrators and motivated the current theoretical investigations.

Stability and error analysis. Our main objective is the derivation of a convergence result for highorder CFQM exponential integrators in the context of non-autonomous linear Schrödinger equations

$$
u^{\prime}(t)=\mathrm{i} H(t) u(t), \quad t \in\left(t_{0}, T\right)
$$

we thereby proceed and complete the investigations in BlAnes, CASAS, THALHAMmER $(2017,2018)$ on the design, numerical comparison, and convergence analysis of CFQM exponential integrators for ordinary and partial differential equations of parabolic type. For the sake of concretion, we provide a detailed stability and local error analysis for the relevant special case, where the Hamilton operator comprises the Laplacian and a regular space-time-dependent potential.

For non-autonomous linear ordinary differential equations, CFQM exponential integrators are composed of exponentials involving linear combinations of certain values of the associated time-dependent matrix; it is straightforward to extend this approach to non-autonomous linear evolution equations of the form (1.1) that are defined by unbounded operators.

An inherent advantage of CFQM exponential integrators over other classes of time integration methods such as Runge-Kutta methods or standard Magnus integrators is that structural properties of the underlying operator family are well-preserved. In essence, this characteristic is explained by the fact that CFQM exponential integrators rely on the solution of Schrödinger equations on certain subintervals $\left[t_{n}, t_{n+1}\right] \subset\left[t_{0}, T\right]$ and involve values of the underlying Hamilton operator at suitably chosen fixed times $t_{\mathrm{fix}} \in\left[t_{n}, t_{n+1}\right]$

$$
v^{\prime}(t)=\mathrm{i} H\left(t_{\mathrm{fix}}\right) v(t), \quad t \in\left(t_{n}, t_{n+1}\right)
$$


thus, it is evident that fundamental properties of the Hamiltonian such as self-adjointness are inherited. On the contrary, higher-order Magnus integrators in addition require the evaluation of commutators of the Hamiltonian at certain times $s_{1}, s_{2} \in\left[t_{n}, t_{n+1}\right]$

$$
\left[H\left(s_{1}\right), H\left(s_{2}\right)\right]=H\left(s_{1}\right) H\left(s_{2}\right)-H\left(s_{2}\right) H\left(s_{1}\right) ;
$$

this leads to evolution equations of a different form, in essence

$$
v^{\prime}(t)=\mathrm{i} H\left(t_{\mathrm{fix}}\right) v(t)+\left[H\left(s_{1}\right), H\left(s_{2}\right)\right] v(t), \quad t \in\left(t_{n}, t_{n+1}\right) .
$$

As illustration, we consider the special case, where the Hamiltonian is given by the second-space derivative and a space-time-dependent potential

$$
H(t)=\partial_{x x}+V(t)
$$

a straightforward calculation implies that the first commutator comprises the first space-derivative

$$
\left[H\left(s_{1}\right), H\left(s_{2}\right)\right] v(t)=\left[\partial_{x x},\left(V\left(s_{2}\right)-V\left(s_{1}\right)\right)\right] v(t)=\partial_{x x}\left(V\left(s_{2}\right)-V\left(s_{1}\right)\right) v(t)+\partial_{x}\left(V\left(s_{2}\right)-V\left(s_{1}\right)\right) \partial_{x} v(t) .
$$

This exemplifies that the underlying Schrödinger equation (1.1) and the evolution equations defining higher-order Magnus integrators (1.2) in general involve differential operators of different nature.

In order to deduce a convergence bound for higher-order Magnus integrators, a suitable approach is to study the associated spatially semi-discrete system and to impose appropriate time stepsize restrictions or, alternatively, to assume higher regularity of the exact solution, see HOCHBRUCK, LUBICH (2003). We note that the second-order exponential mid-point rule is an exceptional case, since it does not involve commutators; it belongs to the class of Magnus integrators as well as to the class of CFQM exponential integrators.

In this work, we confirm the favourable behaviour of CFQM exponential integrators by a rigorous theoretical analysis, which implies unconditional stability in the underlying Hilbert space and the full order of convergence under low regularity requirements on the initial state.

Convenient tools for analysing products of matrix exponentials such as the Baker-CampbellHausdorff formula involve infinite series and thus cannot be applied in connection with unbounded operators; consequently, a certain complexity in the local error analysis of higher-order CFQM exponential integrators for Schrödinger equations is related to an appropriate treatment of compositions of evolution operators. We explore an effective approach that relies on suitable linearisations of the evolution equations satisfied by the exact and numerical solutions, representations by the variation-ofconstants formula, and Taylor series expansions of parts of the integrands; under sufficient regularity requirements on the initial state and the space-time-dependent potential, boundedness of the arising iterated commutators and well-definedness of the resulting local error expansion is ensured.

Outline. The present manuscript has the following structure. In Section 2, we formulate nonautonomous linear Schrödinger equations as abstract evolution equations on Hilbert spaces and specify fundamental assumptions on the defining Hamilton operators. In Section 3, we introduce CFQM exponential integrators and recapitulate second-order and fourth-order example methods. In Section 4, we provide a detailed stability and local error analysis for the special case, where the Schrödinger equation is given by the Laplacian and a regular space-time-dependent potential. In Section 5, we indicate the extension to the general case. 


\section{Class of evolution equations}

Analytical framework. Our analytical framework for a convergence analysis of CFQM exponential integrators applied to Schrödinger equations relies on the theory of self-adjoint operators on Hilbert spaces; for a comprehensive treatment of basic mathematical means and the principles of quantum theory, we refer to BONGAARTS (2015); ENGEL, NAGEL (2000).

Evolution equation. We study the initial value problem for a non-autonomous linear evolution equation of Schrödinger type, cast into the abstract form

$$
\left\{\begin{array}{l}
u^{\prime}(t)=A(t) u(t)=\mathrm{i} H(t) u(t), \quad t \in\left(t_{0}, T\right), \\
u\left(t_{0}\right) \text { given }
\end{array}\right.
$$

throughout, we employ the fundamental assumption that the arising time-dependent Hamilton operator is self-adjoint on the underlying Hilbert space

$$
H(t): \mathscr{D} \subset \mathscr{H} \longrightarrow \mathscr{H}, \quad t \in\left[t_{0}, T\right]
$$

and that the associated evolution operator is unitary

$$
u(t)=\mathscr{E}\left(t-t_{0}, t_{0}\right) u\left(t_{0}\right), \quad\left\|\mathscr{E}\left(t-t_{0}, t_{0}\right)\right\|_{\mathscr{H}}=1, \quad t \in\left[t_{0}, T\right]
$$

Unitarity. For a regular solution to (2.1a), differentiation with respect to time confirms (2.1c); indeed, with the help of the chain rule and due to the required self-adjointness of the Hamilton operator, the following relations implying the unitarity of the evolution operator are obtained

$$
\begin{gathered}
(H(t) u(t) \mid u(t))_{\mathscr{H}}=(u(t) \mid H(t) u(t))_{\mathscr{H}}=\overline{(H(t) u(t) \mid u(t))_{\mathscr{H}}} \in \mathbb{R}, \quad t \in\left[t_{0}, T\right], \\
\frac{\mathrm{d}}{\mathrm{d} t}\|u(t)\|_{\mathscr{H}}^{2}=2 \Re\left(u^{\prime}(t) \mid u(t)\right)_{\mathscr{H}}=2 \Re(\mathrm{i} H(t) u(t) \mid u(t))_{\mathscr{H}}=0, \quad t \in\left(t_{0}, T\right), \\
\|u(t)\|_{\mathscr{H}}^{2}=\left\|u\left(t_{0}\right)\right\|_{\mathscr{H}}^{2}, \quad t \in\left[t_{0}, T\right] .
\end{gathered}
$$

Applications. In view of concrete applications, we primarily study the relevant special case, where the arising Hamilton operator comprises the Laplacian and a space-time-dependent potential, see Section 4; in this situation, it is natural to consider square-integrable complex-valued functions that are defined on the Euclidean space or on a cartesian product of bounded intervals, respectively. That is, the underlying Hilbert space is given by a Lebesgue space

$$
\mathscr{H}=L_{2}(\Omega, \mathbb{C}), \quad \Omega \subseteq \mathbb{R}^{d},
$$

and the common domain of the defining operator family is related to a subspace of a Sobolev space

$$
\mathscr{D} \subseteq H^{2}(\Omega, \mathbb{C})=W_{2}^{2}(\Omega, \mathbb{C})
$$

We point out that in the context of Schrödinger equations generally no additional consistency conditions have to be taken into account. 


\section{Class of time integration methods}

CFQM exponential integrators. Our main objective is to provide a rigorous convergence analysis for commutator-free quasi-Magnus (CFQM) exponential integrators applied to evolution equations of Schrödinger type (2.1). As standard in a time-stepping approach, we choose time grid points with corresponding time stepsizes and determine approximations to the exact solution values by recurrence

$$
\begin{gathered}
t_{0}<\cdots<t_{n}<\cdots<t_{N}=T, \quad \tau_{n}=t_{n+1}-t_{n}, \\
u_{n+1}=\mathscr{S}\left(\tau_{n}, t_{n}\right) u_{n} \approx u\left(t_{n+1}\right)=\mathscr{E}\left(\tau_{n}, t_{n}\right) u\left(t_{n}\right), \quad n \in\{0,1, \ldots, N-1\} ;
\end{gathered}
$$

for a CFQM exponential integrator, the numerical evolution operator is given by the composition of several exponentials that involve linear combinations of certain values of the defining operator

$$
\begin{gathered}
B_{j}\left(\tau_{n}, t_{n}\right)=\sum_{k=1}^{K} a_{j k} A\left(t_{n}+c_{k} \tau_{n}\right), \quad j \in\{1, \ldots, J\}, \\
\mathscr{S}\left(\tau_{n}, t_{n}\right)=\prod_{j=1}^{J} \mathrm{e}^{\tau_{n} B_{j}\left(\tau_{n}, t_{n}\right)}=\mathrm{e}^{\tau_{n} B_{J}\left(\tau_{n}, t_{n}\right)} \ldots \mathrm{e}^{\tau_{n} B_{1}\left(\tau_{n}, t_{n}\right)}, \quad n \in\{0,1, \ldots, N-1\} .
\end{gathered}
$$

A proper choice of the underlying quadrature nodes

$$
c_{k} \in[0,1], \quad k \in\{1, \ldots, K\},
$$

and of the associated real coefficients

$$
a_{j k} \in \mathbb{R}, \quad b_{j}=\sum_{k=1}^{K} a_{j k}, \quad j \in\{1, \ldots, J\}, \quad k \in\{1, \ldots, K\},
$$

permits to attain the desired order of consistency $p \in \mathbb{N}_{\geqslant 1}$; that is, in the context of nonstiff nonautonomous linear ordinary differential equations with associated families of regular time-dependent matrices, an expansion of the local error in the first step with respect to the time stepsize leads to a relation of the form

$$
\mathscr{L}\left(\tau_{0}, t_{0}\right)=\mathscr{S}\left(\tau_{0}, t_{0}\right)-\mathscr{E}\left(\tau_{0}, t_{0}\right)=\mathscr{O}\left(\tau_{0}^{p+1}\right)
$$

Examples. The simplest instance of a second-order CFQM exponential integrator (3.1) is the exponential midpoint rule

$$
\begin{aligned}
& p=2, \quad J=K=1, \quad c_{1}=\frac{1}{2}, \quad a_{11}=b_{1}=1, \\
& \mathscr{S}\left(\tau_{n}, t_{n}\right)=\mathrm{e}^{\tau_{n} A\left(t_{n}+\frac{1}{2} \tau_{n}\right)}, \quad n \in\{0,1, \ldots, N-1\} .
\end{aligned}
$$

The two-stage Gaussian quadrature rule leads to a canonical fourth-order CFQM exponential integrator

$$
\begin{gathered}
p=4, \quad J=K=2, \quad \alpha=\frac{\sqrt{3}}{6}, \quad c_{1}=\frac{1}{2}-\alpha, \quad c_{2}=\frac{1}{2}+\alpha, \\
a_{11}=a_{22}=\frac{1}{4}+\alpha, \quad a_{12}=a_{21}=\frac{1}{4}-\alpha, \quad b_{1}=a_{11}+a_{12}=b_{2}=a_{21}+a_{22}=\frac{1}{2}, \\
B_{j}\left(\tau_{n}, t_{n}\right)=a_{j 1} A\left(t_{n}+c_{1} \tau_{n}\right)+a_{j 1} A\left(t_{n}+c_{2} \tau_{n}\right), \quad j \in\{1,2\}, \\
\mathscr{S}\left(\tau_{n}, t_{n}\right)=\mathrm{e}^{\tau_{n} B_{2}\left(\tau_{n}, t_{n}\right)} \mathrm{e}^{\tau_{n} B_{1}\left(\tau_{n}, t_{n}\right)}, \quad n \in\{0,1, \ldots, N-1\} .
\end{gathered}
$$

For additional examples and detailed information on order conditions, we refer to BLANES, CASAS, THALHAMMER $(2017,2018)$. 
Stability. CFQM exponential integrators (3.1) are composed by terms of the form $\mathrm{e}^{\sigma A(t)}$ for certain values $\sigma \in \mathbb{R}$ and $t \in\left[t_{0}, T\right]$. Within the analytical framework introduced in Section 2, Stone's Theorem implies that any one-parameter group generated by the defining time-dependent linear operator is unitary on the underlying Hilbert space, i.e.

$$
\left\|\mathrm{e}^{\sigma A(t)}\right\|_{\mathscr{H}}=1, \quad \sigma \in \mathbb{R}, \quad t \in\left[t_{0}, T\right],
$$

see ENGEL, NAGEL (2000). By means of this fundamental result, we prove that time integration methods of the format (3.1) and in particular the schemes (3.2) and (3.3) are unconditionally stable, in consistence with the characteristics of the exact evolution operator (2.1), see Section 4.2.

Remark. It is natural to compare CFQM exponential integrators of the form (3.1) to Magnus integrators, which are given by a single exponential involving iterated comutators of certain values of the defining operator, see Blanes, Casas, Oteo, Ros (2009); Blanes, MoAn (2006); Hochbruck, Lubich (2003); Iserles, Kropielnicka, Singh (2019) and references given therein. For both classes of methods, it is ensured that the numerical evolution operator is unitary and hence preserves fundamental properties of the exact evolution operator associated with a Schrödinger equation. As indicated before, for CFQM exponential integrators, this result relies on an application of Stone's Theorem; for a Magnus integrator, it is used that the commutator is skew-Hermitian, which in essence follows from the required self-adjointness of the Hamilton operator

$$
\begin{gathered}
([A(s), A(t)] v \mid w)_{\mathscr{H}}=((A(s) A(t)-A(t) A(s)) v \mid w)_{\mathscr{H}}=-(H(s) H(t) v \mid w)_{\mathscr{H}}+(H(t) H(s) v \mid w)_{\mathscr{H}} \\
\quad=-(H(t) v \mid H(s) w)_{\mathscr{H}}+(H(s) v \mid H(t) w)_{\mathscr{H}}=-(v \mid H(t) H(s) w)_{\mathscr{H}}+(v \mid H(s) H(t) w)_{\mathscr{H}} \\
\quad=(v \mid(A(t) A(s)-A(s) A(t)) w)_{\mathscr{H}}=-(v \mid[A(s), A(t)] w)_{\mathscr{H}} \cdot
\end{gathered}
$$

However, a basic difference between CFQM exponential integrators and Magnus integrators concerns the structure of the associated evolution equations, see the discussion in Section 1 and in particular (1.1) and (1.2). An inherent advantage of CFQM exponential integrators is that the linear combinations arising in (3.1) preserve the structure of the underlying operator family; contrary, a suitable approach for higher-order Magnus integrators relies on considering a spatially semi-discrete setting and imposing major stepsize restrictions or regularity requirements, respectively, see HoCHBRUCK, LUBICH (2003).

Schrödinger versus parabolic equations. In order to ensure the stability of CFQM exponential integrators for non-autonomous linear evolution equations of Schrödinger type, we have to restrict ourselves to schemes involving real coefficients. As an illustration, we consider the Hamilton operator $H(t)=\partial_{x x}+V(t)$, see (1.3) and (4.1); evidently, the property of self-adjointness on the underlying Lebesgue space of square-integrable functions is lost when multiplying by a complex coefficient $a \in \mathbb{C}$, and boundedness of $\mathrm{e}^{\mathrm{i} a H(t)}$ for $t \in\left[t_{0}, T\right]$ cannot be guaranteed. In the context of dissipative quantum systems and parabolic evolution equations, however, it is of interest to study and design CFQM exponential integrators with complex coefficients, see Blanes, CASAS, ThalHAMmer $(2017,2018)$; here, well-definedness of $\mathrm{e}^{a H(t)}$ for $H(t)=\partial_{x x}+V(t)$ or, more generally, for a sectorial operator can be ensured under the assumption $\Re a \geqslant 0$.

\section{Special case}

Situation. In this section, we focus on non-autonomous linear Schrödinger equations, where the Hamilton operator is given by the Laplacian with respect to the spatial variables and a regular real- 
valued space-time-dependent potential acting as multiplication operator

$$
H(t)=\Delta+V(t), \quad V(t)=V(\cdot, t): \Omega \rightarrow \mathbb{R}, \quad t \in\left[t_{0}, T\right]
$$

in view of the abstract formulation (2.1), we again suppress the space dependence. The consideration of this special case is of relevance in applications, see BADER, BLANES, KOPYLOV (2018); HOCHBRUCK, Lubich (2003); Iserles, Kropielnicka, Singh (2019); KyoseVA, Vitanova, Shore (2007) and references given therein.

Notation. With regard to the formulation and derivation of our main result, we henceforth employ convenient abbreviations for Sobolev spaces and related norms

$$
\begin{aligned}
\mathscr{H}^{m} & =H^{m}(\Omega, \mathbb{C})=W_{2}^{m}(\Omega, \mathbb{C}), \quad m \in \mathbb{N}_{\geqslant 0}, \\
\|G\|_{\mathscr{M}^{\ell, m}\left(\left[t_{0}, T\right], \Omega, \mathbb{K}\right)} & =\max _{j \in\{0,1, \ldots, \ell\}}\left\|G^{(j)}(t)\right\|_{L_{\infty}\left(\left[t_{0}, T\right], W_{\infty}^{m}(\Omega, \mathbb{K})\right)}, \quad \ell, m \in \mathbb{N}_{\geqslant 0},
\end{aligned}
$$

where $\mathbb{K}=\mathbb{R}$ or $\mathbb{K}=\mathbb{C}$, respectively; evidently, $\mathscr{H}^{0}$ coincides with the underlying Lebesgue space $\mathscr{H}=L_{2}(\Omega, \mathbb{C})$.

\subsection{Convergence result}

Theoretical result. In the following, we state a theoretical result, which confirms the favourable convergence behaviour of CFQM exponential integrators for evolution equations of Schrödinger type. Compared to other classes of time integration methods, stability is ensured without any restriction on the time stepsizes; we in particular refer to HOCHBRUCK, LUBICH (2003), where stepsize restrictions for Runge-Kutta methods and Magnus integrators are specified in a related context. Moreover, due to the special form of the Hamiltonian, the full order of convergence is obtained under low regularity requirements on the initial state; for Hamilton operators given by more general second-order differential equations, with regard to the convergence result deduced in BLANES, CASAS, THALHAMMER (2018), one would expect the requirement $\left\|u\left(t_{0}\right)\right\|_{\mathscr{H}^{2 p}}<\infty$.

Theorem 1 Assume that the considered non-autonomous linear Schrödinger equation (2.1) is defined by a Hamilton operator of the special structure (4.1). Provided that the arising real-valued space-timedependent potential and the prescribed initial state fulfill suitable regularity requirements such that

$$
C_{V}=\max _{\ell \in\{1, \ldots, p\}}\|V\|_{\mathscr{M}^{\ell, 2(p-\ell)}\left(\left[t_{0}, T\right], \Omega, \mathbb{R}\right)}<\infty, \quad\left\|u\left(t_{0}\right)\right\|_{\mathscr{H}^{p-1}}<\infty
$$

any $p$ th-order CFQM exponential integrator (3.1) satisfies a global error estimate of the form

$$
\begin{gathered}
\left\|u_{N}-u(T)\right\|_{\mathscr{H}} \leqslant\left\|u_{0}-u\left(t_{0}\right)\right\|_{\mathscr{H}}+C \tau_{\max }^{p}\left\|u\left(t_{0}\right)\right\|_{\mathscr{H}^{p-1}}, \\
\tau_{\max }=\max \left\{\tau_{n}: n \in\{0,1, \ldots, N-1\}\right\}
\end{gathered}
$$

the arising constant depends on an upper bound for the time stepsizes, the length of the considered time interval $\left[t_{0}, T\right]$, the bound $C_{V}$, and the method's coefficients, but it is independent of the number of time steps $n$ and the actual time stepsizes.

Proof. Our proof relies on stability bounds and local error estimates, which we deduce in the subsequent Sections 4.2 and 4.3. The constant arising in the local error estimate (4.19) in particular depends on bounds for certain space and time derivatives of the potential on the interval $\left[t_{n}, t_{n+1}\right]$, where 
$n \in\{0,1, \ldots, N-1\}$; estimation by $C_{V}$ leads to a constant that is independent of $n \in\{0,1, \ldots, N-1\}$. Similarly, we estimate the constant in the stability bound (4.2) by a constant that depends on the length of the time interval $\left[t_{0}, T\right]$ and the uniform bound $C_{V}$.

In order to relate the global error to local errors, we employ the telescopic identity

$$
\begin{aligned}
u_{N}-u(T)= & \prod_{n=0}^{N-1} \mathscr{S}\left(\tau_{n}, t_{n}\right)\left(u_{0}-u\left(t_{0}\right)\right) \\
& +\sum_{n=0}^{N-1} \prod_{v=n+1}^{N-1} \mathscr{S}\left(\tau_{v}, t_{v}\right)\left(\mathscr{S}\left(\tau_{n}, t_{n}\right)-\mathscr{E}\left(\tau_{n}, t_{n}\right)\right) \mathscr{E}\left(t_{n}-t_{0}, t_{0}\right) u\left(t_{0}\right) ;
\end{aligned}
$$

due to the fact that the numerical evolution operator associated with a CFQM exponential integrator involving real coefficients is unitary

$$
\left\|\mathscr{S}\left(\tau_{n}, t_{n}\right)\right\|_{\mathscr{H}}=1, \quad n \in\{0,1, \ldots, N-1\},
$$

see (4.3), straightforward estimation in the underlying Hilbert space yields

$$
\left\|u_{N}-u(T)\right\|_{\mathscr{H}} \leqslant\left\|u_{0}-u\left(t_{0}\right)\right\|_{\mathscr{H}}+\sum_{n=0}^{N-1}\left\|\left(\mathscr{S}\left(\tau_{n}, t_{n}\right)-\mathscr{E}\left(\tau_{n}, t_{n}\right)\right) \mathscr{E}\left(t_{n}-t_{0}, t_{0}\right) u\left(t_{0}\right)\right\|_{\mathscr{H}} .
$$

In our expansion of the local error operator with respect to the time stepsize, iterated commutators of the Laplacian and the potential up to order $p-1$ arise naturally and lead to an estimate of the form

$$
\left\|\mathscr{S}\left(\tau_{n}, t_{n}\right)-\mathscr{E}\left(\tau_{n}, t_{n}\right)\right\|_{\mathscr{H} \leftarrow \mathscr{H}^{p-1}} \leqslant C \tau_{n}^{p+1}, \quad n \in\{0,1, \ldots, N-1\},
$$

see (4.19); for this reason, we have to embed the decisive contributions

$$
\left(\mathscr{S}\left(\tau_{n}, t_{n}\right)-\mathscr{E}\left(\tau_{n}, t_{n}\right)\right) \mathscr{E}\left(t_{n}-t_{0}, t_{0}\right) u\left(t_{0}\right), \quad n \in\{0,1, \ldots, N-1\},
$$

in the Sobolev space $\mathscr{H}^{p-1}$. Using the stability bound for the exact evolution operator

$$
\left\|\mathscr{E}\left(t_{n}-t_{0}, t_{0}\right)\right\|_{\mathscr{C}^{p-1}} \leqslant C, \quad n \in\{0,1, \ldots, N-1\},
$$

see (4.2) and (4.6), we obtain the relation

$$
\left\|u_{N}-u(T)\right\|_{\mathscr{H}} \leqslant\left\|u_{0}-u\left(t_{0}\right)\right\|_{\mathscr{H}}+C \sum_{n=0}^{N-1} \tau_{n}^{p+1}\left\|u\left(t_{0}\right)\right\|_{\mathscr{H} p^{p-1}},
$$

which implies the stated global error estimate.

\subsection{Stability}

Stability in Sobolev spaces (Exact evolution operator). The exact solution to the non-autonomous linear Schrödinger equation (2.1) with Hamilton operator given by (4.1) inherits the spatial regularity of the potential and the initial state. As already discussed in Section 2, the evolution operator associated with a self-adjoint Hamilton operator is unitary on the underlying Hilbert space

$$
\left\|\mathscr{E}\left(t-t_{0}, t_{0}\right)\right\|_{\mathscr{H}}=1, \quad t \in\left[t_{0}, T\right]
$$


see (2.1c) and (2.2). Moreover, an estimate of the form

$$
\begin{gathered}
\left\|\mathscr{E}\left(t-t_{0}, t_{0}\right)\right\|_{\mathscr{H}^{m}} \leqslant 1+C_{m}\left(\bar{\tau},\|V\|_{\mathscr{M}^{0, m}\left(\left[t_{0}, t\right], \Omega, \mathbb{R}\right)}\right)\left(t-t_{0}\right) \leqslant C, \\
t-t_{0} \leqslant \bar{\tau}, \quad t \in\left[t_{0}, T\right], \quad m \in \mathbb{N}_{\geqslant 1},
\end{gathered}
$$

holds with a constant that depends on an upper bound for the length of the considered time interval and uniform bounds for certain space derivatives of the potential; the form of the bound and in particular the factor $t-t_{0}$ are essential in view of the derivation of an analogous stability bound for compositions of the numerical evolution operator.

In order to exemplify this result, we consider a non-autonomous linear Schrödinger equation in a single space dimension on the unbounded domain

$$
\partial_{t} \psi(x, t)=\mathrm{i} H(x, t) \psi(x, t)=\mathrm{i}\left(\partial_{x x} \psi(x, t)+V(x, t) \psi(x, t)\right), \quad(x, t) \in \mathbb{R} \times\left(t_{0}, T\right) ;
$$

the first and second spatial derivatives of the solution

$$
\chi_{1}=\partial_{x} \psi, \quad \chi_{2}=\partial_{x x} \psi
$$

satisfy the related evolution equations

$$
\begin{gathered}
\partial_{t} \chi_{m}(x, t)=\mathrm{i}\left(H(x, t) \chi_{m}(x, t)+R_{m}(x, t)\right), \quad m \in\{1,2\}, \\
R_{m}(x, t)=\left\{\begin{array}{ll}
\partial_{x} V(x, t) \psi(x, t) & \text { if } m=1, \\
2 \partial_{x} V(x, t) \chi_{1}(x, t)+\partial_{x x} V(x, t) \psi(x, t) & \text { if } m=2,
\end{array} \quad(x, t) \in \mathbb{R} \times\left(t_{0}, T\right) .\right.
\end{gathered}
$$

A representation by means of the variation-of-constants formula together with the application of the unitarity of the evolution operator (2.1c) leads to the estimate

$$
\begin{aligned}
& \left\|\chi_{1}(\cdot, t)\right\|_{L_{2}(\Omega, \mathbb{C})} \leqslant\left\|\chi_{1}\left(\cdot, t_{0}\right)\right\|_{L_{2}(\Omega, \mathbb{C})}+\int_{t_{0}}^{t}\left\|R_{1}(\cdot, s)\right\|_{L_{2}(\Omega, \mathbb{C})} \mathrm{d} s \\
& \quad \leqslant\left\|\chi_{1}\left(\cdot, t_{0}\right)\right\|_{L_{2}(\Omega, \mathbb{C})}+\left(t-t_{0}\right)\left\|\partial_{x} V\right\|_{L_{\infty}\left(\Omega \times\left[t_{0}, t\right], \mathbb{R}\right)}\left\|\psi\left(\cdot, t_{0}\right)\right\|_{L_{2}(\Omega, \mathbb{C})}, \quad t \in\left[t_{0}, T\right] ;
\end{aligned}
$$

as a consequence, we obtain the relation

$$
\begin{aligned}
& \left\|\chi_{2}(\cdot, t)\right\|_{L_{2}(\Omega, \mathbb{C})} \leqslant\left\|\chi_{2}\left(\cdot, t_{0}\right)\right\|_{L_{2}(\Omega, \mathbb{C})}+\int_{t_{0}}^{t}\left\|R_{2}(\cdot, s)\right\|_{L_{2}(\Omega, \mathbb{C})} \mathrm{d} s \\
& \leqslant\left\|\chi_{2}\left(\cdot, t_{0}\right)\right\|_{L_{2}(\Omega, \mathbb{C})}+2\left(t-t_{0}\right)\left\|\partial_{x} V\right\|_{L_{\infty}\left(\Omega \times\left[t_{0}, t\right], \mathbb{R}\right)} \sup _{s \in\left[t_{0}, t\right]}\left\|\chi_{1}(\cdot, s)\right\|_{L_{2}(\Omega, \mathbb{C})} \\
& \quad+\left(t-t_{0}\right)\left\|\partial_{x x} V\right\|_{L_{\infty}\left(\Omega \times\left[t_{0}, t\right], \mathbb{R}\right)}\left\|\psi\left(\cdot, t_{0}\right)\right\|_{L_{2}(\Omega, \mathbb{C})} \\
& \leqslant\left\|\chi_{2}\left(\cdot, t_{0}\right)\right\|_{L_{2}(\Omega, \mathbb{C})}+2\left(t-t_{0}\right)\left\|\partial_{x} V\right\|_{L_{\infty}\left(\Omega \times\left[t_{0}, t\right], \mathbb{R}\right)}\left\|\chi_{1}\left(\cdot, t_{0}\right)\right\|_{L_{2}(\Omega, \mathbb{C})} \\
& \quad+\left(t-t_{0}\right)\left(2\left(t-t_{0}\right)\left\|\partial_{x} V\right\|_{L_{\infty}\left(\Omega \times\left[t_{0}, t\right], \mathbb{R}\right)}^{2}+\left\|\partial_{x x} V\right\|_{L_{\infty}\left(\Omega \times\left[t_{0}, t\right], \mathbb{R}\right)}\right)\left\|\psi\left(\cdot, t_{0}\right)\right\|_{L_{2}(\Omega, \mathbb{C})}, \quad t \in\left[t_{0}, T\right] .
\end{aligned}
$$

Altogether, this yields the following bounds in the associated Sobolev spaces

$$
\begin{gathered}
\|\psi(\cdot, t)\|_{H^{1}(\Omega, \mathbb{C})} \leqslant\left(1+\left\|\partial_{x} V\right\|_{L_{\infty}\left(\Omega \times\left[t_{0}, t\right], \mathbb{R}\right)}\left(t-t_{0}\right)\right)\left\|\psi\left(\cdot, t_{0}\right)\right\|_{H^{1}(\Omega, \mathbb{C})} \\
\|\psi(\cdot, t)\|_{H^{2}(\Omega, \mathbb{C})} \leqslant\left(1+C_{2}\left(\bar{\tau},\left\|\partial_{x} V\right\|_{L_{\infty}\left(\Omega \times\left[t_{0}, t\right], \mathbb{R}\right)},\left\|\partial_{x x} V\right\|_{L_{\infty}\left(\Omega \times\left[t_{0}, t\right], \mathbb{R}\right)}\right)\left(t-t_{0}\right)\right)\left\|\psi\left(\cdot, t_{0}\right)\right\|_{H^{2}(\Omega, \mathbb{C})} \\
t-t_{0} \leqslant \bar{\tau}, \quad t \in\left[t_{0}, T\right]
\end{gathered}
$$


which are in accordance with (4.2). The extension to higher-order derivatives and higher space dimensions is straightforward.

Stability in Sobolev spaces (Numerical evolution operator). Similar considerations permit to deduce a bound for the numerical evolution operator defining a CFQM exponential integrator (3.1); more precisely, provided that the method's coefficients are real, the analogous relations

$$
\begin{gathered}
\left\|\mathscr{S}\left(\tau_{n}, t_{n}\right)\right\|_{\mathscr{H}}=1, \\
\left\|\mathscr{S}\left(\tau_{n}, t_{n}\right)\right\|_{\mathscr{C}^{m}} \leqslant 1+C_{m}\left(\bar{\tau}_{n},\|V\|_{\mathscr{M}^{0, m}\left(\left[t_{n}, t_{n+1}\right], \Omega, \mathbb{R}\right)}\right) \tau_{n}, \quad \tau_{n} \leqslant \bar{\tau}_{n}, \quad m \in \mathbb{N}_{\geqslant 1}, \\
n \in\{0,1, \ldots, N-1\},
\end{gathered}
$$

hold with a constant that depends on an upper bound for the time stepsize, uniform bounds for certain space derivatives of the potential, and the method's coefficients. By means of the relation $1+x \leqslant \mathrm{e}^{x}$ for $x \geqslant 0$, we obtain a stability estimate for compositions

$$
\begin{aligned}
& \left\|\prod_{n=0}^{N-1} \mathscr{S}\left(\tau_{n}, t_{n}\right)\right\|_{\mathscr{C}^{m}} \leqslant \prod_{n=0}^{N-1} \mathrm{e}^{C_{m}\left(\bar{\tau}_{n},\|V\|_{\mathscr{M}^{0, m}\left(\left[t_{n}, t_{n+1}\right], \Omega, \mathbb{R}\right)}\right) \tau_{n}} \leqslant \mathrm{e}^{C_{m}\left(\bar{\tau},\|V\|_{\mathscr{M}^{0, m}\left(\left[t_{0}, T\right], \Omega, \mathbb{R}\right)}\right)\left(T-t_{0}\right)} \\
& \leqslant C, \quad \max \left\{\bar{\tau}_{n}: n \in\{0,1, \ldots, N-1\}\right\} \leqslant \bar{\tau}, \quad m \in \mathbb{N}_{\geqslant 1}
\end{aligned}
$$

with constant depending in particular on an upper bound for the time stepsizes, the length of the time interval $\left[t_{0}, T\right]$, and uniform bounds for certain space derivatives of the potential.

For a space-time-dependent Hamilton operator of the special structure (4.1), the associated timeindependent operators rewrite as

$$
\begin{gathered}
B_{j}\left(\tau_{n}, t_{n}\right)=\sum_{k=1}^{K} a_{j k} A\left(t_{n}+c_{k} \tau_{n}\right)=\mathrm{i}\left(b_{j} \Delta+\sum_{k=1}^{K} a_{j k} V\left(t_{n}+c_{k} \tau_{n}\right)\right), \\
j \in\{1, \ldots, J\}, \quad n \in\{0, \ldots, N-1\} .
\end{gathered}
$$

If $a_{j k} \in \mathbb{R}$ for any $(j, k) \in\{1, \ldots, J\} \times\{1, \ldots, K\}$, Stone's Theorem ensures unitarity of the arising evolution operators with respect to the norm of the underlying Lebesgue space

$$
\left\|\mathrm{e}^{\sigma B_{j}\left(\tau_{n}, t_{n}\right)}\right\|_{\mathscr{H}}=1, \quad \sigma \in \mathbb{R}, \quad j \in\{1, \ldots, J\}, \quad n \in\{0, \ldots, N-1\},
$$

and thus unconditional stability of the numerical method follows

$$
\left\|\mathscr{S}\left(\tau_{n}, t_{n}\right)\right\|_{\mathscr{H}}=1, \quad n \in\{0, \ldots, N-1\},
$$

see also (3.4) and compare with (4.2). Slight modifications of the arguments used in the exemplification of (4.2) imply the validity of the following estimate on bounded time intervals

$$
\begin{gathered}
\left\|\mathrm{e}^{\sigma B_{j}\left(\tau_{n}, t_{n}\right)}\right\|_{\mathscr{H}^{m}} \leqslant 1+C_{m}\left(\bar{\tau}_{n},\|V\|_{\mathscr{M}^{0, m}\left(\left[t_{n}, t_{n+1}\right], \Omega, \mathbb{R}\right)}\right) \tau_{n}, \quad|\sigma| \leqslant \tau_{n} \leqslant \bar{\tau}_{n}, \\
j \in\{1, \ldots, J\}, \quad n \in\{0,1, \ldots, N-1\}, \quad m \in \mathbb{N}_{\geqslant 1},
\end{gathered}
$$

and justify the stated result (4.3). 


\subsection{Local error}

Approach. In the following, we deduce suitable local error expansions for CFQM exponential integrators (3.1) applied to (2.1), making use of the special structure of the Hamilton operator (4.1). A certain complexity in the treatment of higher-order schemes concerns the correct handling of the arising product of exponentials, since tools that are helpful for matrix exponentials such as the BakerCampbell-Hausdorff $(\mathrm{BCH})$ formula involve infinite series and thus cannot be applied in the context of unbounded operators. Our approach in the spirit of LUNARDI (1995) relies on linearisations of the evolution equations for the exact and numerical solutions as well as representations by the variationof-constants formula; together with Taylor series expansions of parts of the integrands and bounds for naturally arising iterated commutators, the stated result is obtained.

Fourth-order scheme. Due to the fact that the treatment of a general high-order CFQM exponential integrator would involve a considerable amount of technicalities, we provide a rigorous derivation of the local error expansion for the fourth-order scheme based on two Gaussian nodes, see (3.1) and (3.3); it suffices to study the error of (3.3) in the first time step

$$
\begin{gathered}
\tau=\tau_{0}>0, \quad \mathscr{L}\left(\tau, t_{0}\right)=\mathscr{S}\left(\tau, t_{0}\right)-\mathscr{E}\left(\tau, t_{0}\right), \quad \mathscr{S}\left(\tau, t_{0}\right)=\mathrm{e}^{\tau B_{2}\left(\tau, t_{0}\right)} \mathrm{e}^{\tau B_{1}\left(\tau, t_{0}\right)}, \\
B_{j}\left(\tau, t_{0}\right)=\mathrm{i}\left(b_{j} \Delta+a_{j 1} V\left(t_{0}+c_{1} \tau\right)+a_{j 2} V\left(t_{0}+c_{2} \tau\right)\right), \quad j \in\{1,2\}, \\
\alpha=\frac{\sqrt{3}}{6}, \quad c_{1}=\frac{1}{2}-\alpha, \quad c_{2}=\frac{1}{2}+\alpha, \\
a_{11}=a_{22}=\frac{1}{4}+\alpha, \quad a_{12}=a_{21}=\frac{1}{4}-\alpha, \quad b_{1}=a_{11}+a_{12}=b_{2}=a_{21}+a_{22}=\frac{1}{2} .
\end{gathered}
$$

Simplified notation. Henceforth, we specify the arising bounds for space and time derivatives of the potential, but we suppress the dependence of constants on upper bounds for the time stepsize; this slightly simplified notation is also justified by practical implementations, where commonly $\tau \in(0,1)$. For instance, we use that the relation

$$
1+C_{m}\left(\bar{\tau},\|V\|_{\mathscr{M}^{0, m}\left(\left[t_{0}, t_{0}+\tau\right], \Omega, \mathbb{R}\right)}\right) \tau \leqslant C_{m}\left(\|V\|_{\mathscr{M}^{0, m}\left(\left[t_{0}, t_{0}+\tau\right], \Omega, \mathbb{R}\right)}\right)
$$

is valid with generic constant, see (4.2) and (4.3).

Auxiliary abbreviations and estimates. In view of linearisations of the underlying evolution equations for the exact and numerical solutions about the midpoint of the first subinterval and representations by the variation-of-constants formula, we denote

$$
\begin{gathered}
A_{*}=A\left(t_{0}+\frac{\tau}{2}\right)=\mathrm{i}\left(\Delta+V\left(t_{0}+\frac{\tau}{2}\right)\right), \quad R(t)=A(t)-A_{*}, \quad t \in\left[t_{0}, t_{0}+\tau\right], \\
S_{j}\left(\tau, t_{0}\right)=\frac{1}{b_{j}} B_{j}\left(\tau, t_{0}\right)-A_{*}=2\left(a_{j 1} R\left(t_{0}+c_{1} \tau\right)+a_{j 2} R\left(t_{0}+c_{2} \tau\right)\right), \quad j \in\{1,2\} .
\end{gathered}
$$

We make use of the fact that the remainder is determined by values of the potential such that the following auxiliary estimate holds with generic constant

$$
\begin{gathered}
R(t)=\mathrm{i}\left(V(t)-V\left(t_{0}+\frac{\tau}{2}\right)\right) \\
\|R(t)\|_{\mathscr{H}^{m}}+\left\|S_{j}\left(\tau, t_{0}\right)\right\|_{\mathscr{H}^{m}} \leqslant C_{m}\|V\|_{\mathscr{M}^{0, m}\left(\left[t_{0}, t_{0}+\tau\right], \Omega, \mathbb{R}\right)}, \\
t \in\left[t_{0}, t_{0}+\tau\right], \quad j \in\{1,2\}, \quad m \in \mathbb{N}_{\geqslant 0} ;
\end{gathered}
$$


in certain places, to prove that a term has the correct order with respect to the time stepsize, we instead use an integral representation involving the first time derivative

$$
\begin{gathered}
R(t)=\mathrm{i} \int_{t_{0}+\frac{\tau}{2}}^{t} V^{\prime}(\zeta) \mathrm{d} \zeta \\
\|R(t)\|_{\mathscr{H}^{m}}+\left\|S_{j}\left(\tau, t_{0}\right)\right\|_{\mathscr{H}^{m}} \leqslant C_{m}\|V\|_{\mathscr{M}^{1, m}\left(\left[t_{0}, t_{0}+\tau\right], \Omega, \mathbb{R}\right)} \tau \\
t \in\left[t_{0}, t_{0}+\tau\right], \quad j \in\{1,2\}, \quad m \in \mathbb{N}_{\geqslant 0} .
\end{gathered}
$$

Evolution equations and linearisations. The numerical evolution operator associated with (4.5) is composed by two exponentials

$$
\begin{gathered}
\mathscr{S}\left(\tau, t_{0}\right)=\mathscr{S}_{2}\left(\tau, \tau, t_{0}\right) \mathscr{S}_{1}\left(\tau, \tau, t_{0}\right), \\
\mathscr{S}_{j}\left(\sigma, \tau, t_{0}\right)=\mathrm{e}^{\sigma B_{j}\left(\tau, t_{0}\right)}, \quad \sigma \in \mathbb{R}, \quad j \in\{1,2\},
\end{gathered}
$$

which satisfy autonomous linear evolution equations

$$
\begin{gathered}
\frac{\mathrm{d}}{\mathrm{d} \sigma} \mathscr{S}_{j}\left(\sigma, \tau, t_{0}\right)=B_{j}\left(\tau, t_{0}\right) \mathscr{S}_{j}\left(\sigma, \tau, t_{0}\right)=\frac{1}{2} A_{*} \mathscr{S}_{j}\left(\sigma, \tau, t_{0}\right)+\frac{1}{2} S_{j}\left(\tau, t_{0}\right) \mathscr{S}_{j}\left(\sigma, \tau, t_{0}\right), \\
\sigma \in(0, \tau), \quad j \in\{1,2\} .
\end{gathered}
$$

For the exact evolution operator, we employ the analogous identity

$$
\mathscr{E}\left(\tau, t_{0}\right)=\mathscr{E}\left(\frac{\tau}{2}, t_{0}+\frac{\tau}{2}\right) \mathscr{E}\left(\frac{\tau}{2}, t_{0}\right)
$$

and make use of the fact that the associated evolution equation rewrites as

$$
\begin{gathered}
\frac{\mathrm{d}}{\mathrm{d} \sigma} \mathscr{E}\left(\sigma, \vartheta_{0}\right)=A\left(\vartheta_{0}+\sigma\right) \mathscr{E}\left(\sigma, \vartheta_{0}\right)=A_{*} \mathscr{E}\left(\sigma, \vartheta_{0}\right)+R\left(\vartheta_{0}+\sigma\right) \mathscr{E}\left(\sigma, \vartheta_{0}\right), \\
\sigma \in\left(0, \frac{\tau}{2}\right), \quad \vartheta_{0} \in\left\{t_{0}, t_{0}+\frac{\tau}{2}\right\}
\end{gathered}
$$

evidently, a linear transformation yields the equivalent formulation

$$
\begin{gathered}
\frac{\mathrm{d}}{\mathrm{d} \sigma} \mathscr{E}\left(\frac{1}{2} \sigma, \vartheta_{0}\right)= \\
\frac{1}{2} A_{*} \mathscr{E}\left(\frac{1}{2} \sigma, \vartheta_{0}\right)+\frac{1}{2} R\left(\vartheta_{0}+\frac{1}{2} \sigma\right) \mathscr{E}\left(\frac{1}{2} \sigma, \vartheta_{0}\right), \\
\sigma \in(0, \tau), \quad \vartheta_{0} \in\left\{t_{0}, t_{0}+\frac{\tau}{2}\right\} .
\end{gathered}
$$

Auxiliary initial value problem. The evolution equations in (4.8) and (4.9) suggest to study the initial value problem

$$
\left\{\begin{array}{l}
\frac{\mathrm{d}}{\mathrm{d} \sigma} \mathscr{Y}\left(\sigma, Z_{*}, z\right)=Z_{*} \mathscr{Y}\left(\sigma, Z_{*}, z\right)+z(\sigma) \mathscr{Y}\left(\sigma, Z_{*}, z\right), \quad \sigma \in\left(0, \sigma_{0}\right), \\
\mathscr{Y}\left(0, Z_{*}, z\right)=I
\end{array}\right.
$$

and to distinguish the two cases

$$
\left\{\begin{array}{l}
Z_{*}=\frac{1}{2} A_{*}, \quad z=\frac{1}{2} S_{j}\left(\tau, t_{0}\right), \quad j \in\{1,2\}, \quad \sigma_{0}=\tau, \\
Z_{*}=A_{*}, \quad z(\sigma)=R\left(\vartheta_{0}+\sigma\right), \quad \vartheta_{0} \in\left\{t_{0}, t_{0}+\frac{\tau}{2}\right\}, \quad \sigma \in\left[0, \sigma_{0}\right], \quad \sigma_{0}=\frac{\tau}{2} ;
\end{array}\right.
$$


for the sake of clarity, we indicate the dependence of the solution on the defining linear operator and the defining function. Our previous considerations imply relations of the form

$$
\begin{gathered}
\|z(\sigma)\|_{\mathscr{H}^{m}} \leqslant\left\{\begin{array}{l}
C_{m}\|V\|_{\mathscr{M}^{0, m}\left(\left[t_{0}, t_{0}+\tau\right], \Omega, \mathbb{R}\right)}, \quad m \in \mathbb{N}_{\geqslant 0}, \\
C_{m}\|V\|_{\mathscr{M}^{1, m}\left(\left[t_{0}, t_{0}+\tau\right], \Omega, \mathbb{R}\right)} \tau,
\end{array}\right. \\
\left\|\mathrm{e}^{\sigma_{1} Z_{*}}\right\|_{\mathscr{H}}=1, \quad\left\|\mathrm{e}^{\sigma_{1} Z_{*}}\right\|_{\mathscr{H}^{m}} \leqslant C_{m}\left(\|V\|_{\mathscr{M}^{0, m}\left(\left[t_{0}, t_{0}+\tau\right], \Omega, \mathbb{R}\right)}\right), \\
\left\|\mathscr{Y}\left(\sigma, Z_{*}, z\right)\right\|_{\mathscr{H}^{\prime}}=1, \quad\left\|\mathscr{Y}\left(\sigma, Z_{*}, z\right)\right\|_{\mathscr{H}^{m}} \leqslant C_{m}\left(\|V\|_{\mathscr{M}^{0, m}\left(\left[t_{0}, t_{0}+\tau\right], \Omega, \mathbb{R}\right)}\right), \\
\sigma,\left|\sigma_{1}\right| \in\left[0, \sigma_{0}\right], \quad m \in \mathbb{N}_{\geqslant 1},
\end{gathered}
$$

with generic constants, see Section 4.2, and recall (4.6) as well as (4.7).

Solution representation and first expansion. In view of (4.5), we deduce a stepwise expansion of the solution to (4.10). Our starting point is a representation based on the variation-of-constants formula

$$
\begin{gathered}
\mathscr{Y}\left(\sigma_{0}, Z_{*}, z\right)=\mathrm{e}^{\sigma_{0} Z_{*}}+\mathscr{R}_{1}^{(2)}\left(\sigma_{0}, Z_{*}, z\right), \\
\mathscr{R}_{1}^{(2)}\left(\sigma_{0}, Z_{*}, z\right)=\int_{0}^{\sigma_{0}} \mathrm{e}^{\left(\sigma_{0}-\sigma_{1}\right) Z_{*}} z\left(\sigma_{1}\right) \mathscr{Y}\left(\sigma_{1}, Z_{*}, z\right) \mathrm{d} \sigma_{1},
\end{gathered}
$$

involving the exponential and a remainder in integral form. The relations in (4.11) imply that the following bound holds with $p=0$

$$
\left\|\mathscr{R}_{1}^{(p+2)}\left(\sigma_{0}, Z_{*}, z\right)\right\|_{\mathscr{H}} \leqslant C_{0}\left(\|V\|_{\mathscr{M}^{1,0}\left(\left[t_{0}, t_{0}+\tau\right], \Omega, \mathbb{R}\right)}\right) \tau^{p+2},
$$

which explains the role of the upper index. By inserting the analogous identity

$$
\mathscr{Y}\left(\sigma_{1}, Z_{*}, z\right)=\mathrm{e}^{\sigma_{1} Z_{*}}+\int_{0}^{\sigma_{1}} \mathrm{e}^{\left(\sigma_{1}-\sigma_{2}\right) Z_{*}} z\left(\sigma_{2}\right) \mathscr{Y}\left(\sigma_{2}, Z_{*}, z\right) \mathrm{d} \sigma_{2}, \quad \sigma_{1} \in\left[0, \sigma_{0}\right],
$$

into (4.12), we obtain the alternative expansion

$$
\mathscr{Y}\left(\sigma_{0}, Z_{*}, z\right)=\mathrm{e}^{\sigma_{0} Z_{*}}+\mathscr{I}_{1}^{(2)}\left(\sigma_{0}, Z_{*}, z\right)+\mathscr{R}_{1}^{(4)}\left(\sigma_{0}, Z_{*}, z\right) ;
$$

the principal term and the remainder are given by

$$
\begin{gathered}
\mathscr{I}_{1}^{(2)}\left(\sigma_{0}, Z_{*}, z\right)=\mathrm{e}^{\sigma_{0} Z_{*}} \int_{0}^{\sigma_{0}} \mathrm{e}^{-\sigma_{1} Z_{*}} z\left(\sigma_{1}\right) \mathrm{e}^{\sigma_{1} Z_{*}} \mathrm{~d} \sigma_{1}, \\
\mathscr{R}_{1}^{(4)}\left(\sigma_{0}, Z_{*}, z\right)=\mathrm{e}^{\sigma_{0} Z_{*}} \int_{0}^{\sigma_{0}} \int_{0}^{\sigma_{1}} \mathrm{e}^{-\sigma_{1} Z_{*}} z\left(\sigma_{1}\right) \mathrm{e}^{\sigma_{1} Z_{*}} \mathrm{e}^{-\sigma_{2} Z_{*}} z\left(\sigma_{2}\right) \mathscr{Y}\left(\sigma_{2}, Z_{*}, z\right) \mathrm{d} \sigma_{2} \mathrm{~d} \sigma_{1},
\end{gathered}
$$

and fulfill the estimate

$$
\left\|\mathscr{I}_{1}^{(\ell)}\left(\sigma_{0}, Z_{*}, z\right)\right\|_{\mathscr{H}} \leqslant\left\{\begin{array}{l}
C_{0}\left(\|V\|_{\mathscr{M}^{0,0}\left(\left[t_{0}, t_{0}+\tau\right], \Omega, \mathbb{R}\right)}\right) \tau^{\ell} \\
C_{0}\left(\|V\|_{\mathscr{M}^{1,0}\left(\left[t_{0}, t_{0}+\tau\right], \Omega, \mathbb{R}\right)}\right) \tau^{\ell},
\end{array}\right.
$$

with $\ell=2$ as well as (4.13) with $p=2$. In the context of the fourth-order scheme (4.5), we additionally employ the expansion

$$
\mathscr{Y}\left(\sigma_{0}, Z_{*}, z\right)=\mathrm{e}^{\sigma_{0} Z_{*}}+\mathscr{I}_{1}^{(2)}\left(\sigma_{0}, Z_{*}, z\right)+\mathscr{I}_{1}^{(4)}\left(\sigma_{0}, Z_{*}, z\right)+\mathscr{R}_{1}^{(6)}\left(\sigma_{0}, Z_{*}, z\right) ;
$$


the principal term and the remainder

$$
\begin{gathered}
\mathscr{I}_{1}^{(4)}\left(\sigma_{0}, Z_{*}, z\right)=\mathrm{e}^{\sigma_{0} Z_{*}} \int_{0}^{\sigma_{0}} \int_{0}^{\sigma_{1}} \mathrm{e}^{-\sigma_{1} Z_{*}} z\left(\sigma_{1}\right) \mathrm{e}^{\sigma_{1} Z_{*}} \mathrm{e}^{-\sigma_{2} Z_{*}} z\left(\sigma_{2}\right) \mathrm{e}^{\sigma_{2} Z_{*}} \mathrm{~d} \sigma_{2} \mathrm{~d} \sigma_{1}, \\
\mathscr{R}_{1}^{(6)}\left(\sigma_{0}, Z_{*}, z\right)=\mathrm{e}^{\sigma_{0} Z_{*}} \int_{0}^{\sigma_{0}} \int_{0}^{\sigma_{1}} \int_{0}^{\sigma_{2}} \mathrm{e}^{-\sigma_{1} Z_{*}} z\left(\sigma_{1}\right) \mathrm{e}^{\sigma_{1} Z_{*}} \mathrm{e}^{-\sigma_{2} Z_{*}} z\left(\sigma_{2}\right) \mathrm{e}^{\sigma_{2} Z_{*}} \\
\times \mathrm{e}^{-\sigma_{3} Z_{*}} z\left(\sigma_{3}\right) \mathscr{Y}\left(\sigma_{3}, Z_{*}, z\right) \mathrm{d} \sigma_{3} \mathrm{~d} \sigma_{2} \mathrm{~d} \sigma_{1},
\end{gathered}
$$

satisfy (4.15) with $\ell=4$ and (4.13) with $p=4$, respectively. Accordingly, for the local error analysis of a general $p$ th-order CFQM exponential integrator, an auxiliary expansion with remainder fulfilling (4.13) is used.

First expansion for product. In view of (4.8) and (4.9), we specify the expansion obtained for a twofold product

$$
\mathscr{Y}\left(\sigma_{0}, Z_{*}, z_{1}\right) \mathscr{Y}\left(\sigma_{0}, Z_{*}, z_{2}\right)=\mathscr{I}_{2}^{(0)}\left(\sigma_{0}, Z_{*}, z_{1}, z_{2}\right)+\mathscr{R}_{2}^{(6)}\left(\sigma_{0}, Z_{*}, z_{1}, z_{2}\right)
$$

that involves a linear operator $Z_{*}$ and two functions $z_{1}, z_{2}$ satisfying (4.11). A straightforward calculation shows that the principal term is given by

$$
\begin{aligned}
\mathscr{I}_{2}^{(0)}\left(\sigma_{0}, Z_{*}, z_{1}, z_{2}\right)= & \mathrm{e}^{2 \sigma_{0} Z_{*}}+\mathrm{e}^{\sigma_{0} Z_{*}} \mathscr{I}_{1}^{(2)}\left(\sigma_{0}, Z_{*}, z_{2}\right)+\mathscr{I}_{1}^{(2)}\left(\sigma_{0}, Z_{*}, z_{1}\right) \mathrm{e}^{\sigma_{0} Z_{*}} \\
& +\mathrm{e}^{\sigma_{0} Z_{*}} \mathscr{I}_{1}^{(4)}\left(\sigma_{0}, Z_{*}, z_{2}\right)+\mathscr{I}_{1}^{(4)}\left(\sigma_{0}, Z_{*}, z_{1}\right) \mathrm{e}^{\sigma_{0} Z_{*}} \\
& +\mathscr{I}_{1}^{(2)}\left(\sigma_{0}, Z_{*}, z_{1}\right) \mathscr{I}_{1}^{(2)}\left(\sigma_{0}, Z_{*}, z_{2}\right)
\end{aligned}
$$

the remainder comprises several terms

$$
\begin{aligned}
\mathscr{R}_{2}^{(6)}\left(\sigma_{0}, Z_{*}, z_{1}, z_{2}\right)= & \mathscr{R}_{1}^{(6)}\left(\sigma_{0}, Z_{*}, z_{1}\right) \mathscr{Y}\left(\sigma_{0}, Z_{*}, z_{2}\right)+\mathscr{I}_{1}^{(4)}\left(\sigma_{0}, Z_{*}, z_{1}\right) \mathscr{R}_{1}^{(2)}\left(\sigma_{0}, Z_{*}, z_{2}\right) \\
& +\mathscr{I}_{1}^{(2)}\left(\sigma_{0}, Z_{*}, z_{1}\right) \mathscr{R}_{1}^{(4)}\left(\sigma_{0}, Z_{*}, z_{2}\right)+\mathrm{e}^{\sigma_{0} Z_{*}} \mathscr{R}_{1}^{(6)}\left(\sigma_{0}, Z_{*}, z_{2}\right),
\end{aligned}
$$

which have the desired order with respect to the time step size

$$
\left\|\mathscr{R}_{2}^{(6)}\left(\sigma_{0}, Z_{*}, z_{1}, z_{2}\right)\right\|_{\mathscr{H}} \leqslant C_{0}\left(\|V\|_{\mathscr{M}^{1,0}\left(\left[t_{0}, t_{0}+\tau\right], \Omega, \mathbb{R}\right)}\right) \tau^{6},
$$

see (4.13) and (4.15).

Taylor series expansion. With regard to the integrands in (4.14) and (4.16), we perform a Taylor series expansion of a function of the form

$$
\begin{gathered}
g(\sigma)=\mathrm{e}^{-\sigma Z_{*}} G \mathrm{e}^{\sigma Z_{*}}, \quad Z_{*}=\alpha_{1}\left(\Delta+V\left(t_{0}+\frac{\tau}{2}\right)\right), \quad G=\alpha_{2}\left(V(t)-V\left(t_{0}+\frac{\tau}{2}\right)\right), \\
\sigma \in \mathbb{R}, \quad \alpha_{1}, \alpha_{2} \in \mathbb{C}, \quad t \in\left[t_{0}, t_{0}+\tau\right]
\end{gathered}
$$

by means of the following compact notation for iterated commutators

$$
\begin{gathered}
\operatorname{ad}_{Z_{*}}^{0}(G)=G, \quad \operatorname{ad}_{Z_{*}}^{1}(G)=\operatorname{ad}_{Z_{*}}(G)=\left[G, Z_{*}\right]=G Z_{*}-Z_{*} G, \\
\operatorname{ad}_{Z_{*}}^{\ell}(G)=\left[\operatorname{ad}_{Z_{*}}^{\ell-1}(G), Z_{*}\right], \quad \ell \in \mathbb{N}_{\geqslant 2},
\end{gathered}
$$


the values of the function and its derivatives read as

$$
g^{(\ell)}(\sigma)=\mathrm{e}^{-\sigma Z_{*}} \operatorname{ad}_{Z_{*}}^{\ell}(G) \mathrm{e}^{\sigma Z_{*}}, \quad \sigma \in \mathbb{R}, \quad \ell \in \mathbb{N}_{\geqslant 0}
$$

For the convenience of the reader, we recall the Taylor series expansion of order $m \in \mathbb{N}_{\geqslant 0}$ with remainder in integral form

$$
\begin{aligned}
g(\sigma)= & \sum_{\ell=0}^{m} \frac{1}{\ell !}\left(\sigma-\sigma_{*}\right)^{\ell} g^{(\ell)}\left(\sigma_{*}\right) \\
& +\frac{1}{m !}\left(\sigma-\sigma_{*}\right)^{m+1} \int_{0}^{1}(1-\theta)^{m} g^{(m+1)}\left(\sigma_{*}+\theta\left(\sigma-\sigma_{*}\right)\right) \mathrm{d} \theta, \quad \sigma, \sigma_{*} \in \mathbb{R}
\end{aligned}
$$

the specification to (4.18) leads to the relation

$$
\begin{aligned}
& \mathrm{e}^{-\sigma Z_{*}} G \mathrm{e}^{\sigma Z_{*}} \\
& =\mathrm{e}^{-\sigma_{*} Z_{*}}\left(\sum_{\ell=0}^{m} \frac{1}{\ell !}\left(\sigma-\sigma_{*}\right)^{\ell} \operatorname{ad}_{Z_{*}}^{\ell}(G)\right. \\
& \left.\quad+\frac{1}{m !}\left(\sigma-\sigma_{*}\right)^{m+1} \int_{0}^{1}(1-\theta)^{m} \mathrm{e}^{-\theta\left(\sigma-\sigma_{*}\right) Z_{*}} \operatorname{ad}_{Z_{*}^{m+1}}^{m+1}(G) \mathrm{e}^{\theta\left(\sigma-\sigma_{*}\right) Z_{*}} \mathrm{~d} \theta\right) \mathrm{e}^{\sigma_{*} Z_{*}}, \quad \sigma \in \mathbb{R} .
\end{aligned}
$$

Appropriate choices of the center $\sigma_{*} \in \mathbb{R}$ are given below.

Commutators. In order to characterise the regularity requirements that apply accordingly to the above expansion, it remains to study the principal terms in the iterated commutators; as the extension to higher space dimensions is straightforward, it suffices to consider a single space dimension. A simple calculation shows that the first commutator of the Laplacian and a multiplication operator is given by a first-order differential operator involving coefficients that depend on the first and second derivatives of the multiplication operator; more precisely, we have

$$
\begin{gathered}
\left\|\left[\partial_{x x}, V(t)\right] v\right\|_{L_{2}(\Omega, \mathbb{C})} \leqslant 2\left\|\partial_{x} V(t)\right\|_{L_{\infty}(\Omega, \mathbb{R})}\left\|\partial_{x} v\right\|_{L_{2}(\Omega, \mathbb{C})}+\left\|\partial_{x x} V(t)\right\|_{L_{\infty}(\Omega, \mathbb{R})}\|v\|_{L_{2}(\Omega, \mathbb{C})}, \\
v \in H^{1}(\Omega, \mathbb{C}), \quad t \in\left[t_{0}, t_{0}+\tau\right]
\end{gathered}
$$

the second commutator satisfies the relation

$$
\begin{array}{r}
\left\|\left[\partial_{x x},\left[\partial_{x x}, V(t)\right]\right] v\right\|_{L_{2}(\Omega, \mathbb{C})} \leqslant 2\left\|\partial_{x x} V(t)\right\|_{L_{\infty}(\Omega, \mathbb{R})}\left\|\partial_{x x} v\right\|_{L_{2}(\Omega, \mathbb{C})}+4\left\|\partial_{x x x} V(t)\right\|_{L_{\infty}(\Omega, \mathbb{R})}\left\|\partial_{x} v\right\|_{L_{2}(\Omega, \mathbb{C})} \\
+\left\|\partial_{x x x x} V(t)\right\|_{L_{\infty}(\Omega, \mathbb{R})}\|v\|_{L_{2}(\Omega, \mathbb{C})}, \quad v \in H^{2}(\Omega, \mathbb{C}), \quad t \in\left[t_{0}, t_{0}+\tau\right]
\end{array}
$$

By induction, we obtain a bound of the form

$$
\begin{aligned}
\left\|\operatorname{ad}_{Z_{*}}^{m}\left(V(t)-V\left(t_{0}+\frac{\tau}{2}\right)\right)\right\|_{\mathscr{H} \leftarrow \mathscr{H}^{m}} \leqslant\left\{\begin{array}{l}
C_{m}\|V\|_{\mathscr{M}^{0,2 m}\left(\left[t_{0}, t_{0}+\tau\right], \Omega, \mathbb{R}\right)}, \\
C_{m}\|V\|_{\mathscr{M}^{1,2 m}\left(\left[t_{0}, t_{0}+\tau\right], \Omega, \mathbb{R}\right)} \tau,
\end{array}\right. \\
t \in\left[t_{0}, t_{0}+\tau\right], \quad m \in \mathbb{N}_{\geqslant 0},
\end{aligned}
$$

involving a generic constant. 
Expansion of principal term. In order to deduce a suitable representation of the principal term in (4.17), we apply the above stated relations. More precisely, we make use of the following Taylor series expansions

$$
\begin{aligned}
\mathrm{e}^{-\sigma Z_{*}} z(\sigma) \mathrm{e}^{\sigma Z_{*}}= & \mathrm{e}^{-\sigma_{*} Z_{*}} z(\sigma) \mathrm{e}^{\sigma_{*} Z_{*}}+r^{(2)}\left(\sigma, \sigma_{*}, Z_{*}, z\right), \\
= & \mathrm{e}^{-\sigma_{*} Z_{*}}\left(z(\sigma)+\left(\sigma-\sigma_{*}\right) \operatorname{ad}_{Z_{*}}(z(\sigma))+\frac{1}{2}\left(\sigma-\sigma_{*}\right)^{2} \operatorname{ad}_{Z_{*}}^{2}(z(\sigma))\right) \mathrm{e}^{\sigma_{*} Z_{*}} \\
& \quad+r^{(4)}\left(\sigma, \sigma_{*}, Z_{*}, z\right), \quad \sigma \in\left[0, \sigma_{0}\right],
\end{aligned}
$$

with remainders satisfying the bounds

$$
\begin{gathered}
r^{(2)}\left(\sigma, \sigma_{*}, Z_{*}, z\right)=\left(\sigma-\sigma_{*}\right) \int_{0}^{1} \mathrm{e}^{-\left(\sigma_{*}+\theta\left(\sigma-\sigma_{*}\right)\right) Z_{*}} \operatorname{ad}_{Z_{*}}(z(\sigma)) \mathrm{e}^{\left(\sigma_{*}+\theta\left(\sigma-\sigma_{*}\right)\right) Z_{*}} \mathrm{~d} \theta, \\
\left\|r^{(2)}\left(\sigma, \sigma_{*}, Z_{*}, z\right)\right\|_{\mathscr{H} \leftarrow \mathscr{H}^{1}} \leqslant C_{1}\|V\|_{\mathscr{M}^{1,2}\left(\left[t_{0}, t_{0}+\tau\right], \Omega, \mathbb{R}\right)} \tau^{2}, \\
r^{(4)}\left(\sigma, \sigma_{*}, Z_{*}, z\right)=\frac{1}{2}\left(\sigma-\sigma_{*}\right)^{3} \int_{0}^{1}(1-\theta)^{2} \mathrm{e}^{-\left(\sigma_{*}+\theta\left(\sigma-\sigma_{*}\right)\right) Z_{*}} \operatorname{ad}_{Z_{*}}^{3}(z(\sigma)) \mathrm{e}^{\left(\sigma_{*}+\theta\left(\sigma-\sigma_{*}\right)\right) Z_{*}} \mathrm{~d} \theta, \\
\left\|r^{(4)}\left(\sigma, \sigma_{*}, Z_{*}, z\right)\right\|_{\mathscr{H} \leftarrow \mathscr{H}^{3}} \leqslant C_{3}\|V\|_{\mathscr{M}^{1,6}\left(\left[t_{0}, t_{0}+\tau\right], \Omega, \mathbb{R}\right)} \tau^{4}, \quad \sigma \in\left[0, \sigma_{0}\right] .
\end{gathered}
$$

On the one hand, integration implies

$$
\begin{aligned}
\mathscr{I}_{1}^{(2)}\left(\sigma_{0}, \sigma_{*}, Z_{*}, z\right)= & \mathscr{I}_{3}^{(2)}\left(\sigma_{0}, \sigma_{*}, Z_{*}, z\right)+\mathscr{R}_{3}^{(3)}\left(\sigma_{0}, \sigma_{*}, Z_{*}, z\right) \\
= & \mathscr{I}_{3}^{(2)}\left(\sigma_{0}, \sigma_{*}, Z_{*}, z\right)+\mathscr{I}_{3}^{(3)}\left(\sigma_{0}, \sigma_{*}, Z_{*}, z\right)+\mathscr{I}_{3}^{(4)}\left(\sigma_{0}, \sigma_{*}, Z_{*}, z\right) \\
& \quad+\mathscr{R}_{3}^{(5)}\left(\sigma_{0}, \sigma_{*}, Z_{*}, z\right),
\end{aligned}
$$

where the principal terms are given by

$$
\begin{gathered}
\mathscr{I}_{3}^{(2)}\left(\sigma_{0}, \sigma_{*}, Z_{*}, z\right)=\mathrm{e}^{\left(\sigma_{0}-\sigma_{*}\right) Z_{*}} \int_{0}^{\sigma_{0}} z\left(\sigma_{1}\right) \mathrm{d} \sigma_{1} \mathrm{e}^{\sigma_{*} Z_{*}}, \\
\mathscr{I}_{3}^{(3)}\left(\sigma_{0}, \sigma_{*}, Z_{*}, z\right)=\mathrm{e}^{\left(\sigma_{0}-\sigma_{*}\right) Z_{*}} \int_{0}^{\sigma_{0}}\left(\sigma_{1}-\sigma_{*}\right) \operatorname{ad}_{Z_{*}}\left(z\left(\sigma_{1}\right)\right) \mathrm{d} \sigma_{1} \mathrm{e}^{\sigma_{*} Z_{*}}, \\
\mathscr{I}_{3}^{(4)}\left(\sigma_{0}, \sigma_{*}, Z_{*}, z\right)=\mathrm{e}^{\left(\sigma_{0}-\sigma_{*}\right) Z_{*}} \int_{0}^{\sigma_{0}} \frac{1}{2}\left(\sigma_{1}-\sigma_{*}\right)^{2} \operatorname{ad}_{Z_{*}}^{2}\left(z\left(\sigma_{1}\right)\right) \mathrm{d} \sigma_{1} \mathrm{e}^{\sigma_{*} Z_{*}},
\end{gathered}
$$

and the remainders have the desired orders

$$
\begin{gathered}
\mathscr{R}_{3}^{(3)}\left(\sigma_{0}, \sigma_{*}, Z_{*}, z\right)=\mathrm{e}^{\sigma_{0} Z_{*}} \int_{0}^{\sigma_{0}} r^{(2)}\left(\sigma_{1}, \sigma_{*}, Z_{*}, z\right) \mathrm{d} \sigma_{1}, \\
\left\|\mathscr{R}_{3}^{(3)}\left(\sigma_{0}, \sigma_{*}, Z_{*}, z\right)\right\|_{\mathscr{H} \leftarrow \mathscr{H}^{1}} \leqslant C_{1}\|V\|_{\mathscr{M}^{1,2}\left(\left[t_{0}, t_{0}+\tau\right], \Omega, \mathbb{R}\right)} \tau^{3}, \\
\mathscr{R}_{3}^{(5)}\left(\sigma_{0}, \sigma_{*}, Z_{*}, z\right)=\mathrm{e}^{\sigma_{0} Z_{*}} \int_{0}^{\sigma_{0}} r^{(4)}\left(\sigma_{1}, \sigma_{*}, Z_{*}, z\right) \mathrm{d} \sigma_{1}, \\
\left\|\mathscr{R}_{3}^{(5)}\left(\sigma_{0}, \sigma_{*}, Z_{*}, z\right)\right\|_{\mathscr{H} \leftarrow \mathscr{H}^{3}} \leqslant C_{3}\|V\|_{\mathscr{M}^{1,6}\left(\left[t_{0}, t_{0}+\tau\right], \Omega, \mathbb{R}\right)} \tau^{5},
\end{gathered}
$$

see (4.14); on the other hand, we obtain the following representation

$$
\begin{gathered}
\mathscr{I}_{1}^{(4)}\left(\sigma_{0}, \sigma_{*}, Z_{*}, z\right)=\mathscr{I}_{4}^{(4)}\left(\sigma_{0}, \sigma_{*}, Z_{*}, z\right)+\mathscr{R}_{4}^{(5)}\left(\sigma_{0}, \sigma_{*}, Z_{*}, z\right), \\
\mathscr{I}_{4}^{(4)}\left(\sigma_{0}, \sigma_{*}, Z_{*}, z\right)=\mathrm{e}^{\left(\sigma_{0}-\sigma_{*}\right) Z_{*}} \int_{0}^{\sigma_{0}} \int_{0}^{\sigma_{1}} z\left(\sigma_{1}\right) z\left(\sigma_{2}\right) \mathrm{d} \sigma_{2} \mathrm{~d} \sigma_{1} \mathrm{e}^{\sigma_{*} Z_{*}},
\end{gathered}
$$


involving a remainder

$$
\begin{array}{r}
\mathscr{R}_{4}^{(5)}\left(\sigma_{0}, \sigma_{*}, Z_{*}, z\right)=\mathrm{e}^{\sigma_{0} Z_{*}} \int_{0}^{\sigma_{0}} \int_{0}^{\sigma_{1}}\left(r^{(2)}\left(\sigma_{1}, \sigma_{*}, Z_{*}, z\right) \mathrm{e}^{-\sigma_{*} Z_{*}} z\left(\sigma_{2}\right) \mathrm{e}^{\sigma_{*} Z_{*}}\right. \\
\left.+\mathrm{e}^{-\sigma_{1} Z_{*}} z\left(\sigma_{1}\right) \mathrm{e}^{\sigma_{1} Z_{*}} r^{(2)}\left(\sigma_{2}, \sigma_{*}, Z_{*}, z\right)\right) \mathrm{d} \sigma_{2} \mathrm{~d} \sigma_{1}
\end{array}
$$

that fulfills an estimate of the form

$$
\left\|\mathscr{R}_{4}^{(5)}\left(\sigma_{0}, \sigma_{*}, Z_{*}, z\right)\right\|_{\mathscr{H} \leftarrow \mathscr{H}^{1}} \leqslant C_{1}\left(\|V\|_{\mathscr{M}^{1,2}\left(\left[t_{0}, t_{0}+\tau\right], \Omega, \mathbb{R}\right)}\right) \tau^{5}
$$

with generic constant, see (4.11) and (4.16).

Choices of centers. As described below, suitable choices of the centers in the Taylor series expansions permit to extract a common exponential factor; accordingly, we rewrite the expansion (4.17) as

$$
\mathscr{Y}\left(\sigma_{0}, Z_{*}, z_{1}\right) \mathscr{Y}\left(\sigma_{0}, Z_{*}, z_{2}\right)=\mathscr{I}_{5}^{(0)}\left(\sigma_{0}, \zeta, Z_{*}, z_{1}, z_{2}\right)+\mathscr{R}_{5}^{(5)}\left(\sigma_{0}, \zeta, Z_{*}, z_{1}, z_{2}\right)
$$

with principal term given by

$$
\begin{aligned}
& \mathscr{I}_{5}^{(0)}(\left.\sigma_{0}, \zeta, Z_{*}, z_{1}, z_{2}\right) \\
&=\mathrm{e}^{2} \sigma_{0} Z_{*} \\
& \quad+\mathrm{e}^{\sigma_{0} Z_{*}}\left(\mathscr{I}_{3}^{(2)}\left(\sigma_{0}, \zeta_{1}, Z_{*}, z_{2}\right)+\mathscr{I}_{3}^{(3)}\left(\sigma_{0}, \zeta_{1}, Z_{*}, z_{2}\right)+\mathscr{I}_{3}^{(4)}\left(\sigma_{0}, \zeta_{1}, Z_{*}, z_{2}\right)\right) \\
& \quad+\left(\mathscr{I}_{3}^{(2)}\left(\sigma_{0}, \zeta_{2}, Z_{*}, z_{1}\right)+\mathscr{I}_{3}^{(3)}\left(\sigma_{0}, \zeta_{2}, Z_{*}, z_{1}\right)+\mathscr{I}_{3}^{(4)}\left(\sigma_{0}, \zeta_{2}, Z_{*}, z_{1}\right)\right) \mathrm{e}^{\sigma_{0} Z_{*}} \\
& \quad+\mathrm{e}^{\sigma_{0} Z_{*}} \mathscr{I}_{4}^{(4)}\left(\sigma_{0}, \zeta_{3}, Z_{*}, z_{2}\right)+\mathscr{I}_{4}^{(4)}\left(\sigma_{0}, \zeta_{4}, Z_{*}, z_{1}\right) \mathrm{e}^{\sigma_{0} Z_{*}} \\
& \quad+\mathscr{I}_{3}^{(2)}\left(\sigma_{0}, \zeta_{5}, Z_{*}, z_{1}\right) \mathscr{I}_{3}^{(2)}\left(\sigma_{0}, \zeta_{6}, Z_{*}, z_{2}\right) ;
\end{aligned}
$$

the remainder comprises the terms

$$
\begin{aligned}
\mathscr{R}_{5}^{(5)}( & \left.\sigma_{0}, \zeta, Z_{*}, z_{1}, z_{2}\right) \\
= & \mathscr{R}_{2}^{(6)}\left(\sigma_{0}, Z_{*}, z_{1}, z_{2}\right)+\mathrm{e}^{\sigma_{0} Z_{*}} \mathscr{R}_{3}^{(5)}\left(\sigma_{0}, \zeta_{1}, Z_{*}, z_{2}\right)+\mathscr{R}_{3}^{(5)}\left(\sigma_{0}, \zeta_{2}, Z_{*}, z_{1}\right) \mathrm{e}^{\sigma_{0} Z_{*}} \\
& \quad+\mathrm{e}^{\sigma_{0} Z_{*}} \mathscr{R}_{4}^{(5)}\left(\sigma_{0}, \zeta_{3}, Z_{*}, z_{2}\right)+\mathscr{R}_{4}^{(5)}\left(\sigma_{0}, \zeta_{4}, Z_{*}, z_{1}\right) \mathrm{e}^{\sigma_{0} Z_{*}} \\
& \quad+\mathscr{R}_{3}^{(3)}\left(\sigma_{0}, \zeta_{5}, Z_{*}, z_{1}\right) \mathscr{I}_{1}^{(2)}\left(\sigma_{0}, Z_{*}, z_{2}\right)+\mathscr{I}_{3}^{(2)}\left(\sigma_{0}, \zeta_{5}, Z_{*}, z_{1}\right) \mathscr{R}_{3}^{(3)}\left(\sigma_{0}, \zeta_{6}, Z_{*}, z_{2}\right)
\end{aligned}
$$

and thus satisfies an estimate of the form

$$
\left\|\mathscr{R}_{5}^{(5)}\left(\sigma_{0}, \zeta, Z_{*}, z_{1}, z_{2}\right)\right\|_{\mathscr{H} \leftarrow \mathscr{H}^{3}} \leqslant C_{3}\left(\|V\|_{\mathscr{M}^{1,6}\left(\left[t_{0}, t_{0}+\tau\right], \Omega, \mathbb{R}\right)}\right) \tau^{5}
$$

see again (4.11).

Local error expansion. By means of our previous considerations, we obtain a suitable local error expansion for the fourth-order scheme (4.5); more precisely, based on the identity

$$
\begin{aligned}
\mathscr{L}\left(\tau, t_{0}\right)= & \mathscr{S}\left(\tau, t_{0}\right)-\mathscr{E}\left(\tau, t_{0}\right) \\
= & \mathscr{S}_{2}\left(\tau, \tau, t_{0}\right) \mathscr{S}_{1}\left(\tau, \tau, t_{0}\right)-\mathscr{E}\left(\frac{\tau}{2}, t_{0}+\frac{\tau}{2}\right) \mathscr{E}\left(\frac{\tau}{2}, t_{0}\right) \\
= & \mathscr{Y}\left(\tau, \frac{1}{2} A_{*}, \frac{1}{2} S_{2}\left(\tau, t_{0}\right)\right) \mathscr{Y}\left(\tau, \frac{1}{2} A_{*}, \frac{1}{2} S_{1}\left(\tau, t_{0}\right)\right) \\
& \quad-\mathscr{Y}\left(\frac{\tau}{2}, A_{*}, R\left(t_{0}+\frac{\tau}{2}+(\cdot)\right)\right) \mathscr{Y}\left(\frac{\tau}{2}, A_{*}, R\left(t_{0}+(\cdot)\right)\right) \\
= & \mathscr{Y}\left(\tau, \frac{1}{2} A_{*}, \frac{1}{2} S_{2}\left(\tau, t_{0}\right)\right) \mathscr{Y}\left(\tau, \frac{1}{2} A_{*}, \frac{1}{2} S_{1}\left(\tau, t_{0}\right)\right) \\
& \quad-\mathscr{Y}\left(\tau, \frac{1}{2} A_{*}, \frac{1}{2} R\left(t_{0}+\frac{\tau}{2}+\frac{1}{2}(\cdot)\right)\right) \mathscr{Y}\left(\tau, \frac{1}{2} A_{*}, \frac{1}{2} R\left(t_{0}+\frac{1}{2}(\cdot)\right)\right),
\end{aligned}
$$


see (4.8), (4.9), and (4.10), we arrive at the relation

$$
\mathscr{L}\left(\tau, t_{0}\right)=\mathscr{I}_{6}^{(2)}\left(\tau, t_{0}, \zeta, A_{*}, V\right)+\mathscr{R}_{6}^{(5)}\left(\tau, t_{0}, \zeta, A_{*}, V\right) .
$$

The first summand comprises several terms

$$
\begin{aligned}
\mathscr{I}_{6}^{(2)}\left(\tau, t_{0}, \zeta, A_{*}, V\right) \\
=\mathscr{I}_{5}^{(0)}\left(\tau, \zeta, \frac{1}{2} A_{*}, \frac{1}{2} S_{2}\left(\tau, t_{0}\right), \frac{1}{2} S_{1}\left(\tau, t_{0}\right)\right) \\
\quad-\mathscr{I}_{5}^{(0)}\left(\tau, \zeta, \frac{1}{2} A_{*}, \frac{1}{2} R\left(t_{0}+\frac{\tau}{2}+\frac{1}{2}(\cdot)\right), \frac{1}{2} R\left(t_{0}+\frac{1}{2}(\cdot)\right)\right) \\
=\mathscr{I}_{61}^{(2)}\left(\tau, t_{0}, \zeta_{1}, \zeta_{2}, A_{*}, V\right)+\mathscr{I}_{62}^{(2)}\left(\tau, t_{0}, \zeta_{1}, \zeta_{2}, A_{*}, V\right)+\mathscr{I}_{63}^{(2)}\left(\tau, t_{0}, \zeta_{1}, \zeta_{2}, A_{*}, V\right) \\
\quad+\mathscr{I}_{64}^{(4)}\left(\tau, t_{0}, \zeta_{3}, \zeta_{4}, \zeta_{5}, \zeta_{6}, A_{*}, V\right)-\mathscr{I}_{65}^{(4)}\left(\tau, t_{0}, \zeta_{3}, \zeta_{4}, \zeta_{5}, \zeta_{6}, A_{*}, V\right)
\end{aligned}
$$

which have the desired order four, as we justify in detail below; the remainder

$$
\begin{aligned}
\mathscr{R}_{6}^{(5)}\left(\tau, t_{0}, \zeta, A_{*}, V\right)= & \mathscr{R}_{5}^{(5)}\left(\tau, \zeta, \frac{1}{2} A_{*}, \frac{1}{2} S_{2}\left(\tau, t_{0}\right), \frac{1}{2} S_{1}\left(\tau, t_{0}\right)\right) \\
& -\mathscr{R}_{5}^{(5)}\left(\tau, \zeta, \frac{1}{2} A_{*}, \frac{1}{2} R\left(t_{0}+\frac{\tau}{2}+\frac{1}{2}(\cdot)\right), \frac{1}{2} R\left(t_{0}+\frac{1}{2}(\cdot)\right)\right)
\end{aligned}
$$

is well-defined when considered as operator from $\mathscr{H}^{3}$ to $\mathscr{H}$ and satisfies the bound

$$
\left\|\mathscr{R}_{6}^{(5)}\left(\tau, t_{0}, \zeta, A_{*}, V\right)\right\|_{\mathscr{H} \leftarrow \mathscr{H}^{3}} \leqslant C_{3}\left(\|V\|_{\mathscr{M}^{1,6}\left(\left[t_{0}, t_{0}+\tau\right], \Omega, \mathbb{R}\right)}\right) \tau^{5} .
$$

As indicated before, we make use of the fact that the particular choices

$$
\zeta_{1}=0, \quad \zeta_{2}=-\tau, \quad \zeta_{3}=\tau, \quad \zeta_{4}=0, \quad \zeta_{5}=0, \quad \zeta_{6}=\tau,
$$

of the centers in the Taylor series expansions employed for the numerical and exact evolution operators permit to extract common exponential factors. On the one hand, we obtain the relations

$$
\begin{gathered}
\mathscr{I}_{61}^{(2)}\left(\tau, t_{0}, \zeta_{1}, \zeta_{2}, A_{*}, V\right) \\
=\mathrm{e}^{\frac{1}{2} \tau A_{*}}\left(\mathscr{I}_{3}^{(2)}\left(\tau, \zeta_{1}, \frac{1}{2} A_{*}, \frac{1}{2} S_{1}\left(\tau, t_{0}\right)\right)-\mathscr{I}_{3}^{(2)}\left(\tau, \zeta_{1}, \frac{1}{2} A_{*}, \frac{1}{2} R\left(t_{0}+\frac{1}{2}(\cdot)\right)\right)\right) \\
\quad+\left(\mathscr{I}_{3}^{(2)}\left(\tau, \zeta_{2}, \frac{1}{2} A_{*}, \frac{1}{2} S_{2}\left(\tau, t_{0}\right)\right)-\mathscr{I}_{3}^{(2)}\left(\tau, \zeta_{2}, \frac{1}{2} A_{*}, \frac{1}{2} R\left(t_{0}+\frac{\tau}{2}+\frac{1}{2}(\cdot)\right)\right)\right) \mathrm{e}^{\frac{1}{2} \tau A_{*}} \\
=\frac{1}{2} \mathrm{e}^{\tau A_{*}} I_{1}\left(\tau, t_{0}, V\right), \\
I_{1}\left(\tau, t_{0}, V\right)=\int_{0}^{\tau}\left(S_{1}\left(\tau, t_{0}\right)-R\left(t_{0}+\frac{1}{2} \sigma_{1}\right)\right) \mathrm{d} \sigma_{1}+\int_{0}^{\tau}\left(S_{2}\left(\tau, t_{0}\right)-R\left(t_{0}+\frac{\tau}{2}+\frac{1}{2} \sigma_{1}\right)\right) \mathrm{d} \sigma_{1}, \\
\mathscr{I}_{62}^{(2)}\left(\tau, t_{0}, \zeta_{1}, \zeta_{2}, A_{*}, V\right) \\
=\mathrm{e}^{\frac{1}{2} \tau A_{*}}\left(\mathscr{I}_{3}^{(3)}\left(\tau, \zeta_{1}, \frac{1}{2} A_{*}, \frac{1}{2} S_{1}\left(\tau, t_{0}\right)\right)-\mathscr{I}_{3}^{(3)}\left(\tau, \zeta_{1}, \frac{1}{2} A_{*}, \frac{1}{2} R\left(t_{0}+\frac{1}{2}(\cdot)\right)\right)\right) \\
+\left(\mathscr{I}_{3}^{(3)}\left(\tau, \zeta_{2}, \frac{1}{2} A_{*}, \frac{1}{2} S_{2}\left(\tau, t_{0}\right)\right)-\mathscr{I}_{3}^{(3)}\left(\tau, \zeta_{2}, \frac{1}{2} A_{*}, \frac{1}{2} R\left(t_{0}+\frac{\tau}{2}+\frac{1}{2}(\cdot)\right)\right)\right) \mathrm{e}^{\frac{1}{2} \tau A_{*}} \\
=\frac{1}{4} \mathrm{e}^{\tau A_{*}} \operatorname{ad}_{A_{*}}\left(I_{2}\left(\tau, t_{0}, V\right)\right), \\
I_{2}\left(\tau, t_{0}, V\right)=\int_{0}^{\tau} \sigma_{1}\left(S_{1}\left(\tau, t_{0}\right)-R\left(t_{0}+\frac{1}{2} \sigma_{1}\right)\right) \mathrm{d} \sigma_{1}+\int_{0}^{\tau}\left(\sigma_{1}+\tau\right)\left(S_{2}\left(\tau, t_{0}\right)-R\left(t_{0}+\frac{\tau}{2}+\frac{1}{2} \sigma_{1}\right)\right) \mathrm{d} \sigma_{1},
\end{gathered}
$$




$$
\begin{aligned}
& \mathscr{I}_{63}^{(2)}\left(\tau, t_{0}, \zeta_{1}, \zeta_{2}, A_{*}, V\right) \\
& =\mathrm{e}^{\frac{1}{2} \tau A_{*}}\left(\mathscr{I}_{3}^{(4)}\left(\tau, \zeta_{1}, \frac{1}{2} A_{*}, \frac{1}{2} S_{1}\left(\tau, t_{0}\right)\right)-\mathscr{I}_{3}^{(4)}\left(\tau, \zeta_{1}, \frac{1}{2} A_{*}, \frac{1}{2} R\left(t_{0}+\frac{1}{2}(\cdot)\right)\right)\right) \\
& \quad+\left(\mathscr{I}_{3}^{(4)}\left(\tau, \zeta_{2}, \frac{1}{2} A_{*}, \frac{1}{2} S_{2}\left(\tau, t_{0}\right)\right)-\mathscr{I}_{3}^{(4)}\left(\tau, \zeta_{2}, \frac{1}{2} A_{*}, \frac{1}{2} R\left(t_{0}+\frac{\tau}{2}+\frac{1}{2}(\cdot)\right)\right)\right) \mathrm{e}^{\frac{1}{2} \tau A_{*}} \\
& \quad=\frac{1}{16} \mathrm{e}^{\tau A_{*}} \operatorname{ad}_{A_{*}}^{2}\left(I_{3}\left(\tau, t_{0}, V\right)\right), \\
& I_{3}\left(\tau, t_{0}, V\right)=\int_{0}^{\tau} \sigma_{1}^{2}\left(S_{1}\left(\tau, t_{0}\right)-R\left(t_{0}+\frac{1}{2} \sigma_{1}\right)\right) \mathrm{d} \sigma_{1}+\int_{0}^{\tau}\left(\sigma_{1}+\tau\right)^{2}\left(S_{2}\left(\tau, t_{0}\right)-R\left(t_{0}+\frac{\tau}{2}+\frac{1}{2} \sigma_{1}\right)\right) \mathrm{d} \sigma_{1} ;
\end{aligned}
$$

on the other hand, we have

$$
\begin{aligned}
& \mathscr{I}_{64}^{(4)}\left(\tau, t_{0}, \zeta_{3}, \zeta_{4}, \zeta_{5}, \zeta_{6}, A_{*}, V\right) \\
& =\mathrm{e}^{\frac{1}{2} \tau A_{*}} \mathscr{I}_{4}^{(4)}\left(\tau, \zeta_{3}, \frac{1}{2} A_{*}, \frac{1}{2} S_{1}\left(\tau, t_{0}\right)\right)+\mathscr{I}_{4}^{(4)}\left(\tau, \zeta_{4}, \frac{1}{2} A_{*}, \frac{1}{2} S_{2}\left(\tau, t_{0}\right)\right) \mathrm{e}^{\frac{1}{2} \tau A_{*}} \\
& \quad+\mathscr{I}_{3}^{(2)}\left(\tau, \zeta_{5}, \frac{1}{2} A_{*}, \frac{1}{2} S_{2}\left(\tau, t_{0}\right)\right) \mathscr{I}_{3}^{(2)}\left(\tau, \zeta_{6}, \frac{1}{2} A_{*}, \frac{1}{2} S_{1}\left(\tau, t_{0}\right)\right) \\
& =\frac{1}{4} \mathrm{e}^{\frac{1}{2} \tau A_{*}} I_{4}\left(\tau, t_{0}, V\right) \mathrm{e}^{\frac{1}{2} \tau A_{*}}, \\
& I_{4}\left(\tau, t_{0}, V\right) \\
& =\int_{0}^{\tau} \int_{0}^{\sigma_{1}}\left(S_{1}\left(\tau, t_{0}\right) S_{1}\left(\tau, t_{0}\right)+S_{2}\left(\tau, t_{0}\right) S_{2}\left(\tau, t_{0}\right)\right) \mathrm{d} \sigma_{2} \mathrm{~d} \sigma_{1}+\int_{0}^{\tau} S_{2}\left(\tau, t_{0}\right) \mathrm{d} \sigma_{1} \int_{0}^{\tau} S_{1}\left(\tau, t_{0}\right) \mathrm{d} \sigma_{2}, \\
& \mathscr{I}_{65}^{(4)}\left(\tau, t_{0}, \zeta_{3}, \zeta_{4}, \zeta_{5}, \zeta_{6}, A_{*}, V\right) \\
& =\mathrm{e}^{\frac{1}{2} \tau A_{*}} \mathscr{I}_{4}^{(4)}\left(\tau, \zeta_{3}, \frac{1}{2} A_{*}, \frac{1}{2} R\left(t_{0}+\frac{1}{2}(\cdot)\right)\right)+\mathscr{I}_{4}^{(4)}\left(\tau, \zeta_{4}, \frac{1}{2} A_{*}, \frac{1}{2} R\left(t_{0}+\frac{\tau}{2}+\frac{1}{2}(\cdot)\right)\right) \mathrm{e}^{\frac{1}{2} \tau A_{*}} \\
& \quad+\mathscr{I}_{3}^{(2)}\left(\tau, \zeta_{5}, \frac{1}{2} A_{*}, \frac{1}{2} R\left(t_{0}+\frac{\tau}{2}+\frac{1}{2}(\cdot)\right)\right) \mathscr{I}_{3}^{(2)}\left(\tau, \zeta_{6}, \frac{1}{2} A_{*}, \frac{1}{2} R\left(t_{0}+\frac{1}{2}(\cdot)\right)\right) \\
& =\frac{1}{4} \mathrm{e}^{\frac{1}{2} \tau A_{*}} I_{5}\left(\tau, t_{0}, V\right) \mathrm{e}^{\frac{1}{2} \tau A_{*}}, \\
& I_{5}\left(\tau, t_{0}, V\right) \\
& =\quad \int_{0}^{\tau} \int_{0}^{\sigma_{1}}\left(R\left(t_{0}+\frac{1}{2} \sigma_{1}\right) R\left(t_{0}+\frac{1}{2} \sigma_{2}\right)+R\left(t_{0}+\frac{\tau}{2}+\frac{1}{2} \sigma_{1}\right) R\left(t_{0}+\frac{\tau}{2}+\frac{1}{2} \sigma_{2}\right)\right) \mathrm{d} \sigma_{2} \mathrm{~d} \sigma_{1} \\
& \quad+\int_{0}^{\tau} R\left(t_{0}+\frac{\tau}{2}+\frac{1}{2} \sigma_{1}\right) \mathrm{d} \sigma_{1} \int_{0}^{\tau} R\left(t_{0}+\frac{1}{2} \sigma_{2}\right) \mathrm{d} \sigma_{2} .
\end{aligned}
$$

Straightforward Taylor series expansions of the arising integrands lead to the auxiliary estimates

$$
\begin{gathered}
\left\|I_{1}\left(\tau, t_{0}, V\right)\right\|_{\mathscr{H}} \leqslant C_{0}\|V\|_{\mathscr{M}^{4,0}\left(\left[t_{0}, t_{0}+\tau\right], \Omega, \mathbb{R}\right)} \tau^{5}, \\
\left\|\operatorname{ad}_{A_{*}}\left(I_{2}\left(\tau, t_{0}, V\right)\right)\right\|_{\mathscr{H} \leftarrow \mathscr{H}^{1}} \leqslant C_{1}\|V\|_{\mathscr{M}^{3,2}\left(\left[t_{0}, t_{0}+\tau\right], \Omega, \mathbb{R}\right)} \tau^{5}, \\
\left\|\operatorname{ad}_{A_{*}}^{2}\left(I_{3}\left(\tau, t_{0}, V\right)\right)\right\|_{\mathscr{H} \leftarrow \mathscr{H}{ }^{2}} \leqslant C_{2}\|V\|_{\mathscr{M}^{2,4}\left(\left[t_{0}, t_{0}+\tau\right], \Omega, \mathbb{R}\right)} \tau^{5}, \\
\left\|I_{4}\left(\tau, t_{0}, V\right)\right\|_{\mathscr{H}}+\left\|I_{5}\left(\tau, t_{0}, V\right)\right\|_{\mathscr{H}} \leqslant C_{0}\|V\|_{\mathscr{M}^{2,0}\left(\left[t_{0}, t_{0}+\tau\right], \Omega, \mathbb{R}\right)} \tau^{6},
\end{gathered}
$$

see also (4.7), and at once imply

$$
\begin{aligned}
& \left\|\mathscr{I}_{6}^{(2)}\left(\tau, t_{0}, \zeta, A_{*}, V\right)\right\|_{\mathscr{H} \leftarrow \mathscr{H}^{2}} \\
& \quad \leqslant C\left(\|V\|_{\mathscr{M}^{4,0}\left(\left[t_{0}, t_{0}+\tau\right], \Omega, \mathbb{R}\right)},\|V\|_{\mathscr{M}^{3,2}\left(\left[t_{0}, t_{0}+\tau\right], \Omega, \mathbb{R}\right)},\|V\|_{\mathscr{M}^{2,4}\left(\left[t_{0}, t_{0}+\tau\right], \Omega, \mathbb{R}\right)}\right) \tau^{5} .
\end{aligned}
$$


As a consequence, we arrive at the following result

$$
\left\|\mathscr{L}\left(\tau, t_{0}\right)\right\|_{\mathscr{H} \leftarrow \mathscr{H}^{3}} \leqslant C_{3}\left(\max _{\ell \in\{1,2,3,4\}}\|V\|_{\mathscr{M}^{\ell, 2(4-\ell)\left(\left[t_{0}, t_{0}+\tau\right], \Omega, \mathbb{R}\right)}}\right) \tau^{5} .
$$

Local error estimate. Altogether, this proves that the fourth-order CFQM exponential integrator (3.3) satisfies the local error bound

$$
\begin{gathered}
\left\|\mathscr{S}\left(\tau_{n}, t_{n}\right)-\mathscr{E}\left(\tau_{n}, t_{n}\right)\right\|_{\mathscr{H} \leftarrow \mathscr{C}^{p-1}} \leqslant C_{p-1}\left(\max _{\ell \in\{1, \ldots, p\}}\|V\|_{\mathscr{M}^{\ell, 2(p-\ell)}\left(\left[t_{n}, t_{n+1}\right], \Omega, \mathbb{R}\right)}\right) \tau_{n}^{p+1} \leqslant C \tau_{n}^{p+1}, \\
n \in\{0,1, \ldots, N-1\},
\end{gathered}
$$

with $p=4$ and in particular explains the dependence of the constant arising in Theorem 1 on certain space and time derivatives of the potential.

Exponential midpoint rule. It is straightforward to apply the above approach in the context of the exponential midpoint rule; as the scheme involves a single exponential, the calculations are considerably simplified.

\section{General case}

For the sake of completeness, we include the extension of Theorem 1 for Hamilton operators without special structure. The derivation of the local error expansion based on linearisations of the underlying evolution equations, representations by the variation-of-constants formula, and Taylor series expansions of parts of the integrands in principle applies to the general case as well, see Section 4.3; however, for the specification of regularity assumptions on the problem data, more detailed information on the form of the considered Hamiltonians would be required.

Theorem 2 Assume that the operator family defining the non-autonomous linear Schrödinger equation (2.1) and its exact solution are sufficiently regular such that compositions of the form $A^{(\ell)}(s) u^{(m)}(t)$ with $\ell \in\{0,1, \ldots, p\}$ and $m \in\{0,1, \ldots, p-1\}$ remain bounded. Then, any $p$ th-order CFQM exponential integrator (3.1) satisfies a global error estimate of the form

$$
\left\|u_{N}-u(T)\right\|_{\mathscr{H}} \leqslant\left\|u_{0}-u\left(t_{0}\right)\right\|_{\mathscr{H}}+C \tau_{\max }^{p} ;
$$

the arising constant depends on the data of the problem, the length of the considered time interval, and the method's coefficients, but it is independent of the number of time steps $n$ and the maximal time stepsize.

Illustrations. Numerical experiments that confirm the global error bound of Theorem 1 and Theorem 2, respectively, are found in BlAnes, CASAS, ThALHAMmer $(2017,2018)$, see also BADER, Blanes, Kopylov (2018); due to the fact that the exponential midpoint rule is an instance of a Magnus integrator and a $\mathrm{CFQM}$ exponential integrator, we also refer to the numerical example given in HochBRUCK, LUbich (2003). For vanishing dissipation parameter $\delta=0$, the evolution equation considered in Blanes, CASAS, Thalhammer (2017) is of Schrödinger type; the presented results in particular verify the order of convergence and provide numerical evidence that CFQM exponential integrators are superior in efficiency compared to explicit Runge-Kutta methods and Magnus integrators for differential equations involving a higher degree of freedom. 


\section{Acknowledgements}

Part of this work was developed during a research stay at the Wolfgang Pauli Institute Vienna; the authors are grateful to the director Norbert Mauser and the staff members for their support and hospitality. The first three authors acknowledge funding by the Ministerio de Economía y Competitividad (Spain) through project MTM2016-77660-P (AEI/FEDER, UE).

\section{References}

A. Alvermann, H. FehsKe, High-order commutator-free exponential time-propagation of driven quantum systems. J. Comp. Phys. 230/15 (2011) 5930-5956.

A. Alvermann, H. Fehske, P. B. Littlewood, Numerical time propagation of quantum systems in radiation fields. New. J. Phys. 14 (2012) 105008 (22pp).

PH. BAder, S. Blanes, N. Kopylov, Exponential propagators for the Schrödinger equation with a timedependent potential. J. Chem. Phys. 148 (2018) 244109.

S. Blanes, F. CASAs, J. A. Oteo, J. Ros, The Magnus expansion and some of its applications. Physics Reports 470/5-6 (2009) 151-238.

S. Blanes, F. CASAS, M. ThalHAMmer, High-order commutator-free quasi-Magnus exponential integrators for non-autonomous linear evolution equations. Comp. Phys. Commun. 220 (2017) 243-262.

S. Blanes, F. CASAS, M. Thalhammer, Convergence analysis of high-order commutator-free quasi-Magnus exponential integrators for nonautonomous linear evolution equations of parabolic type. IMA J. Numer. Anal. 38/2 (2018) 743-778.

S. Blanes, P. C. MOAN, Fourth- and sixth-order commutator-free Magnus integrators for linear and non-linear dynamical systems. App. Num. Math. 56/12 (2006) 1519-1537.

P. BongaARTs, Quantum Theory. A Mathematical Approach. Springer, Cham, 2015.

K. J. ENGEL, R. NAGEL, One-Parameter Semigroups for Linear Evolution Equations. Springer, New York, 2000.

M. HochbruCK, CH. LUBICH, On Magnus integrators for time-dependent Schrödinger equations. SIAM J. Numer. Anal. 41/3 (2003) 945-963.

A. IsERles, K. KROPIELNICKA, P. Singh, Compact schemes for laser-matter interaction in Schrödinger equation based on effective splittings of Magnus expansion. Comp. Phys. Commun. 234 (2019) 195-201.

E. Kyoseva, N. Vitanova, B. Shore, Physical realization of coupled Hilbert-space mirrors for quantum-state engineering. J. Modern Optics 54 (2007) 2237-2257.

A. Lunard, Analytic Semigroups and Optimal Regularity in Parabolic Problems. Birkhäuser, Basel, 1995. 